1

\title{
The methyl phosphate capping enzyme Bin3 is a stable component of the fission yeast
}

\author{
telomerase holoenzyme
}

\begin{abstract}
Jennifer Porat ${ }^{1}$, Moaine El Baidouri ${ }^{2,3}$, Jorg Grigull ${ }^{4}$, Jean-Marc Deragon ${ }^{2,3,5}$, \& Mark A.
\end{abstract}
Bayfield $^{1^{*}}$

1. Department of Biology, York University, Toronto, Canada

2. LGDP-UMR5096, Université de Perpignan Via Domitia, Perpignan, France

3. CNRS LGDP-UMR5096, Perpignan, France

4. Department of Mathematics and Statistics, York University, Toronto, Canada

5. Institut Universitaire de France, Paris, France

*Correspondence: bayfield@yorku.ca (M.A.B.)

\section{Abstract:}

The telomerase holoenzyme is critical for maintaining eukaryotic genome integrity. In addition

to a reverse transcriptase and an RNA template, telomerase contains additional proteins that

protect the telomerase RNA and promote holoenzyme assembly. Here we report that the

methyl phosphate capping enzyme (MePCE) Bin3 is a stable component of the S. pombe

telomerase holoenzyme. Bin3 associates with the telomerase and the U6 snRNA through an

interaction with the recently described LARP7 family member Pof8, and we demonstrate that

these two factors are evolutionarily linked in fungi. Our data suggest that the association of

Bin3 with telomerase is independent of its methyltransferase activity, but rather that Bin3

negatively regulates TER1 levels and telomere length. Taken together, this work yields new 
24 insight into the composition, assembly, and regulation of the telomerase holoenzyme in fission

25 yeast as well as the breadth of its evolutionary conservation.

\section{Introduction:}

28 To ensure complete DNA replication, the termini of eukaryotic chromosomes contain tandem

29 repeats, or telomeres, that can be continually extended as the ends of linear chromosomes are

30 lost through DNA replication. Telomeres serve as protection from DNA degradation, end-to-end

31 fusions, chromosomal rearrangements, and chromosome loss ${ }^{1,2}$. With very few exceptions,

32 eukaryotes extend telomeric DNA sequences through the telomerase holoenzyme, a complex

33 containing the telomerase reverse transcriptase (Trt1 in the fission yeast Schizosaccharomyces

34 pombe), an RNA template (TER1 in S. pombe), and accessory proteins that promote complex

35 assembly and tethering to telomeric DNA ${ }^{1-4}$. While the reverse transcriptase/RNA template

36 core of telomerase is generally well-conserved among eukaryotes, major differences exist,

37 including in the sequence and structure of the RNA template. In contrast to ciliate telomerase

38 RNAs, which are small RNA Polymerase III transcripts ${ }^{5}$, yeast and metazoan telomerase RNAs

39 are longer and transcribed by RNA Polymerase II as precursors. These precursors then undergo

40 an extensive maturation process to yield the mature form that integrates into the telomerase

41 holoenzyme $3,4,6,7$.

43 In S. pombe, the mature form of TER1 is generated from an intron-containing precursor through

44 a spliceosome-catalyzed reaction involving release of the $5^{\prime}$ exon prior to exon ligation ${ }^{8}$. TER1

45 maturation then proceeds with the sequential binding of the Sm and Lsm complexes. Sm 
proteins, well-characterized for their role in splicing, bind TER1 immediately upstream the 5'

47 splice site and promote 3' maturation and the addition of a 5' trimethylguanosine (TMG) cap by

48 the methyltransferase Tgs $1^{9}$. The Sm complex is then replaced by the Lsm2-8 complex, which

49 serves to protect the mature 3' end of TER1 from exonucleolytic degradation and promotes the

50 interaction between TER1 and Trt1 in the active telomerase holoenzyme. The switch from the

51 Sm to Lsm complexes correlates with distinct, non-overlapping substrates: TER1 precursors are

52 exclusively bound by the Sm complex, while the mature form of TER1, ending in a polyuridylate

53 stretch upstream the spliceosomal cleavage site, is only bound by the Lsm2-8 complex ${ }^{9}$.

55 More recently, a role has been proposed for Pof8, a La-related protein 7 (LARP7) homolog, in

56 telomerase assembly and telomere maintenance in S. pombe. Ciliate LARP7 family proteins,

57 including p65 from Tetrahymena thermophila, have previously been characterized for their

58 functions in telomerase assembly ${ }^{10}$. Binding of p65 to stem IV of the $T$. thermophila telomerase

59 RNA TER results in a conformational change to the RNA and subsequent binding of the reverse

60 transcriptase TERT, suggesting a p65-dependent hierarchical assembly of the telomerase

61 holoenzyme ${ }^{11}$. Pof8 is hypothesized to function similarly in S. pombe, with Pof8 binding to TER1

62 promoting recruitment of the Lsm2-8 complex and enhancing the interaction between TER1

63 and Trt1. Accordingly, Pof8 deletion strains possess shorter telomeres indicative of a defect in

64 telomerase function ${ }^{12-14}$. Pof8 also functions in telomerase RNA quality control through

65 recognition of the correctly folded pseudoknot region and subsequent recruitment of the Lsm2-

668 complex to protect the $3^{\prime}$ end from degradation ${ }^{15}$. Telomerase-related functions in LARP7

67 family proteins, particularly Pof8, map to a conserved C-terminal domain characteristic of 
LARP7 family members, the extended RNA recognition motif (xRRM). The Pof8 xRRM is a major determinant for Pof8-mediated telomerase activity, contributing to TER1 binding and complex assembly ${ }^{13,16}$. As other fungal Pof8 homologs also possess an xRRM, it is anticipated that Pof8 may have a conserved role in telomere maintenance beyond fission yeast ${ }^{13,17}$.

73 Conversely, LARP7 homologs in higher eukaryotes are best characterized in the transcriptional

74 regulatory 7SK snRNP, which includes the RNA polymerase III transcribed 7SK snRNA, the

75 methyl phosphate capping enzyme (MePCE), and hexamethylene bisacetamide-inducible protein (HEXIM1/2). The 7SK snRNP inhibits transcription by sequestering positive transcription

77 elongation factor ( $\mathrm{P}-\mathrm{TEFb}$ ) and preventing it from phosphorylating the C-terminal domain of the

78 largest subunit of RNA Polymerase II, which is associated with the transition into productive

79 transcription elongation ${ }^{18-20}$. The La module and XRRM of LARP7 bind 7SK through its

80 polyuridylate trailer and the $3^{\prime}$ hairpin, respectively, and promote MePCE recruitment to the

81 complex ${ }^{21,22}$. In addition to adding a $\gamma$-monomethyl phosphate cap (i.e. $\mathrm{CH}_{3}-\mathrm{pppN}$ ) to 7SK

82 snRNA as a means of protecting it from $5^{\prime}$ exonucleolytic degradation, MePCE remains stably

83 bound to 7SK snRNA to stabilize the complex ${ }^{21}$. MePCE, a homolog of the Drosophila

84 melanogaster Bin3/BCDIN3 protein, also catalyzes the addition of this atypical cap structure to

85 U6 snRNA ${ }^{23}$. Bin3/MePCE homologs are present in many eukaryotes, but to date little has been

86 studied outside of humans and Drosophila ${ }^{24}$. More recent work has identified BCIND3D, a

87 related Bin3 family protein overexpressed in breast cancer cells, as the enzyme responsible for

88 methylating the $5^{\prime}$ monophosphate of histidine transfer RNA and pre-miR-145 25,26. Despite the 
many insights into the role of Bin3/MePCE in RNA processing in higher eukaryotes, the function of the essential S. pombe Bin3 homolog has yet to be determined ${ }^{27,28}$.

92 In this work, we have explored the RNA targets of the S. pombe Bin3 homolog in an effort to

93 uncover its function. In addition to an expected association of Bin3 with the U6 snRNA, we

94 present data showing that Bin3 is a stable component of the S. pombe telomerase RNP. Our

95 results also provide evidence for an evolutionarily conserved interaction between Bin3 and LARP7 homologs. Importantly, we show that the Bin3-Pof8 interaction is required for the

97 recruitment of Bin3 to both the U6 snRNA and the active telomerase holoenzyme. Additionally,

98 we provide evidence that Bin3 does not catalyze the addition of a $\gamma$-monomethyl phosphate

99 cap on TER1, suggesting a catalytic independent function. Rather, we present evidence that

101 increases in steady-state levels of TER1 and longer telomeres. Taken together, our results

102 identify Bin3 as a novel component of the S. pombe telomerase holoenzyme, thus adding a new 103 layer of complexity to telomerase assembly and telomere maintenance.

\section{Results:}

\section{S. pombe Bin3 is essential and interacts with the U6 snRNA and the telomerase RNA TER1}

107 While Bin3/MePCE is conserved in many eukaryotes, its presence in the fission yeast S. pombe,

108 an organism lacking 7SK snRNA ${ }^{24,29}$, is not well understood. Unlike the longer Bin3/MePCE

109 homologs from higher eukaryotes, S. pombe Bin3 only contains a conserved 
111 pombe Bin3 to that of $H$. sapiens MePCE and D. melanogaster Bin3 reveals that previously

112 identified residues critical for SAM and SAH binding and nucleotide binding ${ }^{30}$ are highly

113 conserved in S. pombe (Figure S1A,B). To validate genome-wide deletion screens identifying

114 Bin3 as an essential fission yeast protein ${ }^{27,28}$, we ectopically expressed GST-tagged Bin3 cDNA

115 on the thiamine repressible pRep4x plasmid in a wild type strain and subsequently knocked out

116 the chromosomal copy (Figure S1C). Counter-selection of the Bin3-expressing plasmid on 5'FOA

117 resulted in lethality of the bin $3 \Delta$ strain, with no phenotype for a wild type strain, confirming its

118 essential nature. Furthermore, overexpression of GST-Bin3 in wild-type or bin3 $\Delta$ cells (i.e. in the

119 absence of thiamine) resulted in very slow growth, compared to normal growth when GST-Bin3

120 expression was repressed by addition of thiamine. Growth in the absence or presence of added

121 thiamine had no effect on cells transformed with an empty vector (Figure S1D). These data

122 thus suggest that in addition to Bin3 being essential, its overexpression is poorly tolerated in S.

123 pombe.

124

125 We next sought to determine the RNA substrates of S. pombe Bin3 to better understand its role 126 in the processing of fission yeast non-coding RNA(s). Following integration of a protein A (PrA)

127 tag into the Bin3 genomic locus, we performed RIP-Seq and plotted Bin3 IP reads against the IP

128 from an untagged strain, followed by validation of potential candidate substrates with northern

129 blotting (Figure 1, S2, Table S1). Consistent with previous reports of MePCE interacting with U6

130 snRNA ${ }^{23}$, U6 emerged as one of the most highly enriched Bin3-interacting RNA transcripts. S.

131 pombe U6 is an unusual transcript in that it is transcribed by RNA Polymerase III yet contains an 132 mRNA-type intron removed by the spliceosome ${ }^{31,32}$. Alignment of RIP-Seq reads to the S. 
133 pombe genome suggested that Bin3 interacts exclusively with the spliced form of U6. We

134 confirmed this with northern blotting, demonstrating robust immunoprecipitation of mature

135 U6 and no immunoprecipitation of the intron-containing precursor (Figure 1B). Chromatin

136 immunoprecipitation studies suggest that 7SK and U6 snRNA may be co-transcriptionally

137 modified by the human MePCE homolog ${ }^{33}$, however our data are consistent with a model

138 wherein S. pombe Bin3 interacts with U6 post-splicing.

139

140 Surprisingly, the S. pombe telomerase RNA TER1 was also highly enriched in Bin3

141 immunoprecipitates (Figure 1A, C). Given the previously established link between the human

142 Bin3 homolog MePCE and human LARP7, ${ }^{20}$ as well as the established link between the $S$.

143 pombe LARP7 homolog Pof8 and the telomerase holoenzyme ${ }^{12-14}$, we considered the

144 possibility that Bin3 may also be part of the telomerase holoenzyme through an evolutionarily

145 conserved interaction with LARP7 family members.

147 Bin3 interacts with the mature form of TER1

149 Since TER1 processing and maturation involves a spliceosome-catalyzed reaction ${ }^{8}$, we first set

150 out to determine the processing state of Bin3-associated TER1 by sequencing the $5^{\prime}$ and $3^{\prime}$ ends

151 through circularized rapid amplification of cDNA ends (Figure 2A). We also performed cRACE on

152 TER1 immunoprecipitated by Pof8, the telomerase reverse transcriptase Trt1, and Lsm3, all

153 known components of the mature TER1-containing active telomerase holoenzyme ${ }^{3,4,9,12-14}$. All

154 sequenced candidates had a discrete $5^{\prime}$ end consistent with the reported $5^{\prime}$ end ${ }^{3,4}$ and a similar 
155 distribution of $3^{\prime}$ ends ending immediately upstream the $5^{\prime}$ splice site ${ }^{8}$, suggesting that Bin3

156 interacts with the full-length, 3' end-matured TER1 associated with the active telomerase

157 holoenzyme. The heterogeneity observed at the $3^{\prime}$ end of all candidates is likely the result of

158 exonucleolytic nibbling prior to binding of the Lsm2-8 complex ${ }^{9}$. To investigate this further, we

159 subjected TER1 RNA immunoprecipitated by Bin3, Trt1, Pof8 and Lsm3 to RNase H cleavage to

160 generate shorter 5' and 3' fragments and compared fragment size by northern blot (Figure 2B).

161 The sizes of the $5^{\prime}$ and $3^{\prime}$ ends of TER1 were similar across immunoprecipitates, further

162 substantiating that Bin3 binds the mature form of TER1, rather than the intron-containing

163 precursor.

165 Further TER1 processing in S. pombe involves the addition of a 5' trimethylguanosine (TMG) cap

166 by $\operatorname{Tgs} 1^{9}$. Since Bin3 homologs are normally associated with the formation of a $\gamma$-monomethyl

167 phosphate cap, and to further understand when Bin3 interacts with TER1 with respect to TER1

168 processing, we examined the cap structure of Bin3-associated TER1 by immunoprecipitating

169 RNA associated with Bin3 and subsequently re-immunoprecipitating this RNA with an anti-TMG

170 antibody. The specificity of the anti-TMG antibody was confirmed by showing that it effectively

171 enriched the TMG cap containing U5 snRNA over the TMG cap lacking U6 snRNA or an

172 unrelated tRNA (Figure 2C). While Bin3-associated U6 snRNA was not immunoprecipitated by

173 the anti-TMG antibody, in agreement with data demonstrating the presence of a $y$-monomethyl

174 phosphate cap ${ }^{34}$, Bin3-associated TER1 RNA was effectively enriched by anti-TMG

175 immunoprecipitation. These data are consistent with previous work demonstrating a TMG cap

176 on TER1 in S. pombe, and also with Bin3 associating with TER1 following spliceosomeal cleavage 
177 and $5^{\prime}$ TMG capping, similar to what has been posited for Pof ${ }^{12}$. This suggests that Bin3

178 interacts with the primary cohort of TER1 transcripts, and not a $\mathrm{y}$-monomethyl phosphate

179 capped subset, and that TER1 is not a substrate for Bin3-catalyzed modification.

\section{Bin3 interacts with components of the mature telomerase holoenzyme}

182 Since our results indicate that Bin3 interacts with the mature form of TER1, we set out to

183 confirm the presence of Bin3 in the mature S. pombe telomerase holoenzyme. We

184 immunoprecipitated Bin3 and identified interacting proteins through mass spectrometry,

185 followed by validation using co-immunoprecipitation (Figure 3). We identified Pof8, the Lsm2-8

186 complex, and Trt1 in Bin3 immunoprecipitates, all of which make up the catalytically active core

187 telomerase holoenzyme ${ }^{9,12-14,35-37}$ (Table S2, S3). We also detected the RNase P and RNase

188 MRP subunit Pop100 in Bin3 immunoprecipitations. Pop1, Pop6, and Pop7, subunits of RNase P

189 and RNase MRP in budding yeast, have recently been identified as components of the budding

190 yeast telomerase complex ${ }^{38-40}$, suggesting that the involvement of RNase P and RNase MRP

191 subunits in telomere maintenance is evolutionarily conserved. The finding that Bin3 interacts

192 with core components of the telomerase holoenzyme, and not the Sm complex nor the TMG

193 capping enzyme Tgs1, suggests Bin3 does indeed associate with the active telomerase

194 holoenzyme rather than a precursor. The involvement of Bin3 in the telomerase holoenzyme

195 was further substantiated by gene ontology analysis to determine overrepresented biological

196 processes and cellular components among the top 50 Bin3 interactors. Telomerase holoenzyme

197 complex assembly and telomere maintenance via telomerase emerged as the top

198 overrepresented biological processes (Figure 3A). Similarly, overrepresented cellular 
components include the Lsm2-8 complex, the telomerase holoenzyme, and spliceosomal snRNPs (Table S4). We also repeated immunoprecipitations in the presence of benzonase to determine if these interactions are direct or mediated through a nucleic acid intermediate

202 (Table S2). With the exception of Pof8, interactions between Bin3 and other components of the telomerase holoenzyme were lost, indicative of an interaction mediated by TER1.

205 We confirmed the interactions between Bin3 and Pof8, as well as Bin3 and Trt1, through co-

206 immunoprecipitation (Figures 3B, 3C, S3). We also performed co-immunoprecipitations with

207 benzonase, demonstrating that the interaction between Bin3 and Pof8 persists with benzonase

208 treatment, while the Bin3-Trt1 interaction is lost (Figures 3B and 3C). The apparent nucleic acid-

209 dependence of the interaction between Bin3 and the majority of the telomerase holoenzymes

210 is reminiscent both of the budding yeast telomerase RNA TLC1, which acts as a scaffold to

211 nucleate telomerase holoenzyme assembly ${ }^{41}$, as well as the MePCE/Bin3-containing 7SK snRNP

212 42. The finding that Bin3 and Pof8 interact directly is in agreement with previous reports

213 demonstrating a protein-protein interaction between MePCE and LARP7 in the context of the

214 vertebrate 7SK snRNP ${ }^{33,42}$, suggesting the direct interaction may be evolutionarily conserved.

216 The apparent immunodepletion of Trt1 during Bin3 immunoprecipitation (Figure 3C, see

217 flowthrough lanes) indicated an absence of detectable Trt1-containing telomerase

218 holoenzymes lacking Bin3, substantiating the idea that Bin3 is a stoichiometric component of

219 the S. pombe telomerase holoenzyme. Consistent with this, benzonase treatment prior to Bin3

220 immunoprecipitation resulted in reappearance of Trt1 in the immunoprecipitation flowthrough. 
221 On the other hand, affinity purification of Bin3 did not completely deplete Pof 8 from the Bin3

222 immunoprecipitation flow through, consistent with either Bin3-independent functions for Pof8

223 or with possible dissociation of Pof8 from some Bin3-containing complexes (see U6, below)

224 (Figure 3B).

225

Bin3 is recruited to the active telomerase holoenzyme through the LARP7 family protein Pof8

227 We then tested whether Bin3 was recruited to the telomerase holoenzyme through its

228 interaction with Pof8, much like the human LARP7 homolog promotes MePCE recruitment to

229 7SK snRNA ${ }^{21}$. Immunoprecipitation of Bin3 in the context of a pof8D strain resulted in a

230 complete loss of TER1 binding, providing evidence that the interaction between Bin 3 and TER1

231 is dependent on an interaction between Bin3 and Pof8 and Pof8 and TER1 (Figure 4A).

232 Unexpectedly, the interaction between Bin3 and U6 was also lost in the pof8 . Recent findings

233 indicate that human LARP7 binds U6 to guide post-transcriptional modification ${ }^{43,44}$, so it is

234 tempting to speculate that Pof8 may also mediate Bin3 binding to U6 in fission yeast. To

235 address this, we immunoprecipitated Pof8 alongside Bin3 and other components of the

236 telomerase holoenzyme and looked for U6 enrichment. We observed slight enrichment of U6 in

237 Pof8-myc immunoprecipitates relative to immunoprecipitation using an untagged strain or the

238 Trt1-myc tagged strain (Figure 4B). We did not see enrichment for the intron-containing

239 precursor, suggesting that like Bin3, Pof8 interacts with the spliced form of U6. The slight

240 enrichment of U6 with a Pof8 immunoprecipitation, compared to the more robust

241 immunoprecipitation seen with Bin3 and Lsm3, suggests that Pof8 may transiently bind U6,

242 perhaps serving to load Bin3 on U6. Since LARP7 binding has been reported to disrupt the 
243 catalytic activity of MePCE ${ }^{33}$, a transient Pof8-U6 interaction may be consistent with U6

244 receiving a $y$-monomethyl phosphate cap, compared to TER1, where stable Pof8 binding may

245 prevent Bin3 catalytic activity.

247 Knowing that Bin3 interacts with TER1 and components of the telomerase holoenzyme, we next

248 set out to confirm whether Bin3 is part of the catalytically active telomerase holoenzyme. We

249 performed a previously described in vitro telomerase assay that relies on the presence of the

250 TER1- and Trt1-containing telomerase holoenzyme to extend an oligonucleotide resembling

251 telomeric DNA ${ }^{12}$. Bin3 immunoprecipitates extended the oligonucleotide in a similar or greater

252 manner to Trt1 and Pof8 immunoprecipitates, as well as showed the same loss of activity upon

253 RNase A treatment, supporting the idea that Bin3, much like Pof8, is a component of the active

254 telomerase holoenzyme (Figure 4C). Consistent with our previous results, we also observed a

255 loss of activity for Bin3 immunoprecipitated from a pof8 $\Delta$ strain, providing another line of

256 evidence that Bin3 is recruited to the telomerase holoenzyme through Pof8. Since Trt1 is only

257 recruited to the telomerase holoenzyme following Pof8 and Lsm2-8 binding, coupled with the

258 idea that the assay relies on the presence of a reverse transcriptase, these results are

259 consistent with a model in which Bin3 is recruited to TER1 through its interaction with Pof8 and

260 remains bound through subsequent holoenzyme assembly and the catalytic cycle (Figure 4D).

261

262 Evidence that Pof8 is required for telomerase holoenzyme assembly and maintenance of

263 telomere length ${ }^{12-14}$, as well as for Bin3 recruitment to telomerase, prompted the question of

264 whether we could rescue the short telomere phenotype of a pof $8 \Delta$ strain by overexpressing 
265 Bin3. Consistent with a model in which Bin3 only assembles with the active telomerase

266 holoenzyme through its interaction with Pof8, Bin3 overexpression in a pof8 $\Delta$ strain was

267 insufficient to rescue telomere shortening and the resultant chromosome fusions occurring

268 after telomeres reach a critically short length ${ }^{12}$ (Figure S4).

\section{Bin3 negatively regulates TER1 accumulation and telomere lengthening}

271 Following the finding that Bin3 is a component of the S. pombe telomerase holoenzyme, we

272 wondered at the function of Bin3, and how it might interface with the stabilization and complex

273 assembly roles previously described for Pof8 ${ }^{12-15}$. As Bin3 deletion is lethal and its

274 overexpression results in a slow growth phenotype (Figure S1D), we varied Bin3 expression by

275 expressing plasmid-encoded Bin3 under the $n m t 1$ promoter in bin $3 \Delta$ cells grown in rich media

276 containing thiamine. Under these conditions, we determined that thiamine-repressed, plasmid

277 encoded Bin3 was expressed approximately two-fold lower than Bin3 endogenously expressed

278 from the genomic locus (Figure 5A). Surprisingly, considering Pof8's role in TER1 stabilization,

279 we found this lower expression of Bin3 relative to wild type cells resulted in increased steady-

280 state levels of TER1 (Figure 5B). To determine the effects of Bin3 depletion on telomere length,

281 we continually passaged bin $3 \Delta$ strains in minimal media containing supplemented thiamine and

282 visualized telomeres by southern blotting (Figure 5C). We observed a small but noticeable

283 telomere lengthening relative to a wild type strain, as well as the disappearance of a lower

284 band. Taken together, our results suggest that Bin3 may act as a negative regulator to limit

285 TER1 levels and telomerase activity under wild type conditions, in contrast to the stabilizing

286 roles of its binding partner Pof8. 
Pof8-like proteins are associated with Bin3-like proteins in diverse fungal lineages

The interaction between Bin3 and Pof8, coupled with the conservation of such an interaction in other examined eukaryotes, led us to wonder at the extent of this interaction on a phylogenetic scale. While Bin3/MePCE and LARP7/Pof8 family members are ubiquitous in higher eukaryotes,

292 their absence or presence is more varied in fungal lineages. We queried hundreds of

293 representative fungal species for the presence of Bin3- and Pof8-like proteins and identified

294 many species with a) both a Bin3 and Pof8 homolog, b) only a Bin3 homolog, or c) neither a

295 Bin3 nor a Pof8 homolog (Figure 6, S5). Conversely, only four out of 472 examined species

296 contained a Pof8-like protein but no Bin3-like protein, and in these species the Pof8 homolog

297 was noted to have a shortened N-terminal domain that would lack the La module observed in

298 other LARP7 family members, raising the possibility that members of this rare cohort may not

299 be bona-fide LARP7 family members, at least as these are currently appreciated ${ }^{17}$. Based on

300 the observed distribution, it seems likely that both genes were present in a fungi common

301 ancestor, but Pof8 or both Bin3 and Pof8 were lost in certain lineages. Examination of the

302 distribution of Bin3 and LARP7-family members in basal eukaryotic lineages reveals a similar

303 pattern: Bin3 can be present without LARP7, but there are no instances where a LARP7-family

304 member exists in a lineage lacking a Bin3 homolog (Table S9). Similarly, LARP7-family proteins

305 are represented in Alveolates, Stramenopiles, Amoebozoa, Fungi, and Metazoans, while Bin3-

306 family proteins are present in all eukaryotic lineages. Since the presence of Pof8/LARP7 is very

307 highly linked to the presence of a Bin3 homolog, these data suggest that the function of LARP7 
308 family members may be more intimately associated with the function of Bin3/MePCE than has

309 been previously appreciated.

\section{Discussion:}

312 In this study, we have identified Bin3 as a novel component of the S. pombe telomerase

313 holoenzyme. In addition to showing an interaction between Bin3 and U6 snRNA, we

314 demonstrate that Bin3 interacts with the telomerase RNA TER1 and components of the active

315 telomerase holoenzyme in a manner that is dependent on the presence of the LARP7 family

316 protein Pof8. Together, our results indicating that Bin3 interacts with the mature, spliced form

317 of TER1 and Bin3 immunoprecipitates possess telomerase activity in vitro strongly suggest that

318 Bin3 is a constitutive component of the telomerase holoenzyme containing Trt1, Pof8, Lsm2-8,

319 and Est1 scaffolded on TER1 (Figure 4D). The idea of Pof8 and Bin3 scaffolded on TER1 is

320 reminiscent of LARP7 and MePCE in the 7SK snRNP, with Pof8/LARP7 recruiting Bin3/MePCE to

321 the RNA substrate. Similar to our data showing Pof8 recruiting Bin3 to TER1 and the telomerase

322 holoenzyme, LARP7 has also been demonstrated to recruit MePCE to the 7SK snRNP in human

323 cells ${ }^{21}$. Thus, the protein-protein interaction and subsequent recruitment to RNA substrates by

324 LARP7 appears evolutionarily conserved in fission yeast and higher eukaryotes. We also present

325 data demonstrating that the presence of Pof8-like proteins is nearly universally correlated with

326 the presence of Bin3-like proteins in fungi, suggesting that the Bin3-Pof8 interaction is highly

327 conserved and more prevalent than previously anticipated. Since Pof8 is required to load Bin3

328 onto the telomerase RNA and U6 SnRNA in fission yeast, this phylogenetic distribution raises 
the question of whether Bin3 homologs in fungi lacking a LARP7 homolog have evolved a mechanism to bind RNA targets in a Pof8/LARP7 independent fashion.

332 Since TER1 undergoes several processing steps before assembly into the telomerase

333 holoenzyme, we were able to assess TER1 processing state to determine when Bin3 interacts

334 with TER1 with respect to the timing of TER1 maturation. We show that Bin3-associated TER1

335 has a mature $3^{\prime}$ end, indicative of spliceosome-catalyzed end processing, and a 5' TMG cap. This

336 is consistent with previously reported data on the timing of Pof8 binding; since our data

337 indicates that the interaction between Bin3 and TER1 is dependent on Pof8, the most

338 parsimonious explanation is that Pof8, and thus Bin3, bind TER1 following 3' maturation and

339 TMG capping, preceding the binding of the Lsm2-8 complex ${ }^{12}$. We show that the interaction

340 between Bin3 and TER1 is dependent on Pof8, leading to the conclusion that Bin3 interacts with

341 TER1 at the same stage as Pof8, resulting in the hierarchical assembly of the active telomerase

342 holoenzyme.

344 The finding that Bin3-associated TER1 is immunoprecipitated by a TMG antibody argues against

345 a catalytic role for Bin3 in TER1 processing. Our data, coupled with previous work characterizing

346 the cap structure on the mature TER1 bound to Trt1 ${ }^{3,4}$, supports the idea that Bin3 has a

347 capping-independent function in the telomerase holoenzyme. LARP7 binding to MePCE in the

348 7SK snRNP inhibits its capping activity; MePCE, in turn, promotes the interaction between 7SK

349 and LARP7, thereby stabilizing the complex ${ }^{33}$. In the S. pombe telomerase holoenzyme and U6

350 snRNP, we can hypothesize that Pof8 may also inhibit the methyltransferase activity of Bin3 by 
351 binding to and obscuring the active site. This is consistent with our results suggesting a

352 transient interaction between Pof8 and U6 that may function to load Bin3 on U6. In such a

353 model, an interaction between Bin3 and Pof8 is necessary to recruit Bin3 to U6 and following

354 dissociation of Pof8, the catalytic site of Bin3 would become available to cap U6.

356 Additionally, our work identifying Bin3 as a telomerase-associated protein brings to mind work

357 identifying a box H/ACA motif at the $3^{\prime}$ end of vertebrate telomerase RNA ${ }^{45}$. Box H/ACA RNAs

358 form an RNP complex with Dyskerin/Cbf5, Nop10, Nhp2, and Gar1 to guide noncoding RNA

359 pseudouridylation ${ }^{46,47}$. Although Dyskerin is a pseudouridine synthase, to date no evidence has

360 pointed to a modification function for Dyskerin in the context of telomerase RNA. Rather, the

361 Dyskerin-containing H/ACA complex has a structural role in telomerase biogenesis, with binding

362 of the H/ACA RNP to telomerase RNA leading to a conformational change in the RNA that

363 promotes $3^{\prime}$ processing and maturation over exosome-mediated decay ${ }^{48}$. This is reminiscent of

364 the apparent catalytic independent role we observed for Bin3 in fission yeast telomerase,

365 although our data point towards Bin3 having a role in limiting TER1 accumulation, perhaps as a

366 negative regulator of telomere elongation.

Bin3 and Pof8 having seemingly opposing functions in TER1 maturation and the telomerase RNP

370 are lethal. Assuming Bin3 functions in limiting telomerase activity by decreasing TER1 levels, a

371 lack of Bin3 negative regulation may be detrimental, much like the shelterin component Tpz1

372 and the telomere cap complex subunit Stn1, both of which are negative regulators of 
373 telomerase and essential proteins in S. pombe ${ }^{27,49}$. In contrast, since Pof8 promotes telomerase

374 activity, its deletion doesn't result in unchecked telomere lengthening. It remains to be found

375 how exactly Bin3 limits TER1 accumulation but it is possible that Bin3 interfaces with decay

376 pathways, such as the established exosome-mediated 3' decay pathway ${ }^{12,14}$ or an as yet

377 undiscovered pathway analogous to the $5^{\prime}$ decay of human telomerase RNA ${ }^{50}$. Bin3 may

378 therefore be important for maintaining telomere homeostasis by decreasing TER1 levels under

379 conditions where cell cycle progression or telomere lengthening are unfavorable.

381 Another facet of this work builds on the idea that components of the telomerase holoenzyme

382 are more conserved than previously appreciated. In addition to the ubiquitous telomerase

383 reverse transcriptase, previous work has identified LARP7 family proteins in both ciliates and

384 yeast ${ }^{10,12-14}$, arguing a level of evolutionary conservation. Other recent findings implicate the

385 human LARP7 homolog in telomere maintenance ${ }^{51}$, suggesting this conservation may extend to

386 higher eukaryotes. We now also provide evidence indicating the presence of RNase P and

387 RNase MRP subunits in fission yeast, much like the RNase P and RNase MRP subunits found in

388 the budding yeast telomerase complex ${ }^{38-40}$. The presence of RNase $\mathrm{P}$ and RNase MRP subunits

389 in two divergent yeast species supports the importance of these factors in the function of a

390 breadth of telomerase holoenzymes.

391

392 Our results indicating that Bin3 is part of the telomerase holoenzyme were quite unexpected,

393 as Bin3 has been well-characterized in higher eukaryotes for its role in the processing,

394 maturation, and protection of RNA Polymerase III transcripts ${ }^{23,33}$. An intriguing idea involves 
polymerase switching through telomerase RNA evolution. Ciliate telomerase RNA is transcribed by RNA Polymerase III and subsequently binds the LARP7 homolog p65 through the terminal

397 polyuridylate stretch common to RNA Polymerase III transcripts ${ }^{10,52}$. Since MePCE and LARP7

398 also associate with RNA Polymerase III transcripts, it is possible that the Pof8-bound RNA

399 Polymerase II transcribed TER1, and the S. pombe telomerase RNP as a whole, represent an

400 intermediate step in the evolution of telomerase RNA between ciliates and the RNA Polymerase

401 II transcribed telomerase RNA of higher eukaryotes. Future work should investigate the

402 presence of Bin3/MePCE homologs in the telomerase holoenzymes of other species,

403 particularly in ciliates, which possess both an RNA Polymerase III transcribed telomerase RNA

404 and a LARP7 homolog. Such findings will continue to develop emerging ideas regarding

405 conservation in telomerase RNA processing and the composition of telomerase RNPs.

407 Acknowledgements:

408 We thank Toru Nakamura and Virginia Zakian for sharing published yeast strains and Marc

409 Fabian and Raymund Wellinger for helpful comments on the manuscript. J.P. is supported by a

410 Canada Graduate Scholarship (Doctoral) from the National Sciences and Engineering Research

411 Council of Canada. M.A.B. is supported by a Discovery Grant from NSERC ("Impact of chemical

412 modification of non-coding RNAs on gene expression in S. pombe"). JMD is supported by the

413 Institut Universitaire de France (IUF), JMD and MEB by the CNRS and the University of

414 Perpignan (UPVD) and their study is set within the framework of the "Laboratoires

415 d'Excellences (LABEX)" TULIP (ANR-10-LABX-41) and of the "École Universitaire de Recherche 416 (EUR)" TULIP-GS (ANR-18-EURE-0019). 
419 Author contributions:

420 J.P. conceived of the study, designed, performed, and analyzed all experiments, and wrote the

421 first draft of the manuscript with input from all authors. M.E.B. and J-M.D. performed

422 phylogenetic analysis. J.G. and J.P. analyzed RNA-Seq data. M.A.B. conceived of the study,

423 designed experiments, supervised the project, and edited the manuscript.

425 Materials and Methods:

426 Strains, constructs, and growth media

427 Strains were grown in yeast extract with supplements (YES) or Edinburgh Minimal Media

428 (EMM), as indicated. Expression of genes under the control of the $n m t 1$ promoter was

429 repressed with the addition of $15 \mu \mathrm{M}$ thiamine. Tag integration and knockouts were generated

430 with a previously described PCR-based strategy and verified by PCR and western blotting ${ }^{53}$

431 (primer sequences in Table S6). Protein A-tagged strains were generated according to ${ }^{54}$.

432 Correct genotypes were selected on YES plates with the corresponding antibiotic ( $200 \mu \mathrm{g} / \mathrm{mL}$

433 G418, Sigma; $100 \mu \mathrm{g} / \mathrm{mL}$ Nourseothricin, GoldBio; $100 \mu \mathrm{g} / \mathrm{mL}$ phleomycin, Invivogen). Other

434 strains were created by mating and antibiotic selection. A list of strains is provided in Table S5.

$436 \quad$ Native protein extract and immunoprecipitation

437 S. pombe cells were grown in YES at $30^{\circ} \mathrm{C}$ to mid-log phase, harvested, and subject to cryogenic

438 disruption using a mortar and pestle. Cell powder was lysed in $50 \mathrm{mM} \mathrm{NaCl}, 20 \mathrm{mM}$ Hepes pH 
7.4, 55 mM KOAc, 0.5\% Triton X, 0.1\% Tween-20, 0.2 mM PMSF, 1:100 Protease Inhibitor

Cocktail (Thermo, 78430), and $0.004 \mathrm{U} / \mu \mathrm{L}$ RNase inhibitor (Invitrogen, AM2694). For Protein A-

441 tagged strains, immunoprecipitation was carried out with Rabbit IgG-conjugated (MP-

442 Biomedicals, SKU 085594) Dynabeads (Invitrogen, 14301) as described ${ }^{55}$. Myc-tagged proteins

443 were immunoprecipitated with Protein G Dynabeads (Invitrogen, 10003D) coated with anti-myc

444 antibody (Sigma, M4439). Beads were washed 4 times with $400 \mu$ L lysis buffer. For RNA

445 immunoprecipitations, bound RNA was isolated by treatment of beads with $0.1 \%$ SDS and 0.2

$446 \mathrm{mg} / \mathrm{mL}$ Proteinase $\mathrm{K}$ (Sigma, $\mathrm{P} 2308$ ) at $37^{\circ} \mathrm{C}$ for 30 minutes, followed by extraction with phenol:

447 chloroform:isoamyl alcohol (25:24:1) and ethanol precipitation.

$449 \quad$ RNA preparation and Northern blotting

450 Total RNA was isolated from native protein extracts by incubation with $0.5 \%$ SDS, $0.2 \mathrm{mg} / \mathrm{mL}$

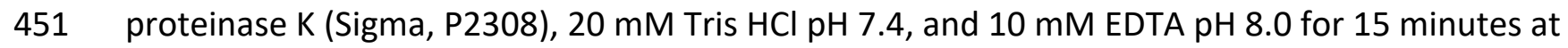

$45250^{\circ} \mathrm{C}$, followed by phenol: chloroform extraction and ethanol precipitation. Northern blot

453 analysis was performed as described using 8\% TBE-urea polyacrylamide gels ${ }^{56}$. Briefly,

454 electrophoresed RNA was transferred to positively charged nylon membranes (Perkin Elmer,

455 NEF988001) with the iBlot 2 system (Thermo, IB21001) and probed with ${ }^{32} \mathrm{P} \gamma$-ATP-labeled DNA

456 probes (probe sequences provided in Table S7). For RNase H digestion, RNA was incubated with

$45725 \mathrm{pmol}$ RNase $\mathrm{H}$ probe (provided in Table S7) for 5 minutes at $65^{\circ} \mathrm{C}$ and slow cooled to $37^{\circ} \mathrm{C}$,

458 followed by digestion with $5 \mathrm{U}$ RNase $\mathrm{H}$ (NEB, M02975) for 30 minutes at $37^{\circ} \mathrm{C}$. The reaction

459 was stopped with the addition of $25 \mu \mathrm{M}$ EDTA pH 8.0 and phenol: chloroform extraction and 
460 ethanol precipitation. TMG-capped RNAs were isolated from RNase H-treated

461 immunoprecipitates with an $\alpha-$ TMG agarose conjugate (Sigma, NA02A), according to ${ }^{4}$.

462

$463 \underline{\text { qRT-PCR }}$

$4641 \mu \mathrm{g}$ Turbo DNase-treated RNA was reverse transcribed with the iScript cDNA synthesis kit

465 (BioRad, 1708890), treated with $0.5 \mu \mathrm{L}$ RNase cocktail (Invitrogen, AM2286), and diluted 1:10.

466 cDNA was quantified using the SensiFAST SYBR No-Rox kit (Bioline, BIO-98005) and $1 \mu \mathrm{M}$ of

467 each primer (primer sequences provided in Table S7). qPCR settings were as follows: $95^{\circ} \mathrm{C}$ for

46810 minutes and 40 cycles consisting of 10 seconds at $95^{\circ} \mathrm{C}, 20$ seconds at $60^{\circ} \mathrm{C}$, and 20 seconds

469 at $72^{\circ} \mathrm{C}$, followed by melting curve analysis. Bin3 $m R N A$ levels were normalized to his $1 \mathrm{mRNA}$

470 levels and subsequently subject to paired two-tailed student's $t$-tests ( $n=3$ biological replicates,

471 each with 6 technical replicates).

472

$473 \quad$ RNA Seq and RIP Seq

474 Libraries were constructed from immunoprecipitated RNA samples and rRNA-depleted total

475 RNA samples by the TCAC Sequencing Facility at the Hospital for Sick Children in Toronto.

476 Libraries were sequenced on an Illumina Hi-Seq 2500 for 50-bp single-end reads. Reads were

477 mapped to the S. pombe ASM294v2 genome using kallisto ${ }^{57}$ using parameters -single -

478 fragment-length $=50-s d=5$ and subsequently visualized on IGV ${ }^{58}$. Reads were filtered to

479 include transcripts containing 4 or more reads in at least one of the Bin3 immunoprecipitated

480 replicates and TPM values were subject to median-normalization. Gaussian mixture modeling

481 (GMM) was applied to calculate the probability of an interaction using log of odds ${ }^{59}$. Reads 
482 were further filtered to exclude transcripts with a log of odds score less than 3.17. The log of

483 the average of median-normalized TPM values from the Bin3 immunoprecipitates was plotted

484 against that of the control immunoprecipitations.

485

486 Circularized Rapid Amplification of cDNA Ends (cRACE)

487 Immunoprecipitated RNA was treated with 2 U TURBO DNase (Invitrogen, AM2239) and

488 dephosphorylated with $1 \mathrm{U}$ calf intestinal alkaline phosphatase (NEB, M0290), followed by

489 decapping and circularization with RNA 5' Pyrophosphohydrolase (NEB, M0356S) and T4 RNA

490 Ligase 1 (NEB, M0204S). TER1 was amplified from circularized RNA with the OneStep RT-PCR Kit

491 (Qiagen, 210210) (primer sequences in Table S7). cRACE products were cloned into the pGEM-T

492 vector (Promega, A1360) and sequenced by the TCAG DNA Sequencing Facility at the Hospital

493 for Sick Children in Toronto.

494

$495 \quad$ Mass spectrometry and GO analysis

496 Half of the native protein extract was pre-treated with $0.625 \mathrm{U} / \mu \mathrm{L}$ Benzonase (Millipore, E1014)

497 at $37^{\circ} \mathrm{C}$ for 30 minutes. The reaction was stopped with $5 \mathrm{mM}$ EDTA pH 8.0 and Protein A

498 immunoprecipitation was performed in the same manner as for RNA immunoprecipitations.

499 After washes with lysis buffer, beads were washed with $0.1 \mathrm{M} \mathrm{NH}_{4} \mathrm{OAc}$ and $0.1 \mathrm{mM} \mathrm{MgCl} 2$ and

500 eluted with $0.5 \mathrm{M} \mathrm{NH}_{4} \mathrm{OH}$ for 20 minutes at room temperature. Eluates were lyophilized and

501 subject to in-solution tryptic digestion. LC-MS/MS analysis was performed by the IRCM

502 Proteomics Discovery Platform on a Q Exactive HF. 
504 Raw data was processed and analyzed using the MaxQuant software package 1.5.1.2 ${ }^{60}$ and the

505 fission yeast UP000002485 reference proteome (24/11/2019). Settings used for MaxQuant

506 analysis were as reported ${ }^{61}$. Proteins present in immunoprecipitations from both biological

507 replicates and absent from control immunoprecipitations were considered genuine Bin3

508 interacting partners and ranked by peak intensity. PANTHER overrepresentation tests (GO

509 Ontology database released 2019-12-09) were conducted on the top 50 Bin3 interacting

510 partners. Fisher's exact tests with FDR correction were performed using the GO biological

511 process complete and GO cellular component complete data sets.

513 Co-immunoprecipitation and western blotting

514 Cells were harvested at mid-log phase, resuspended in lysis buffer $(10 \mathrm{mM}$ Tris $\mathrm{HCl} \mathrm{pH} 7.5,150$

$515 \mathrm{mM} \mathrm{NaCl}, 0.5 \% \mathrm{NP40}, 1: 100$ Protease Inhibitor Cocktail), and lysed with acid-washed glass

516 beads (Sigma, G8772). Immunoprecipitations were carried out as described and proteins were

517 eluted by resuspending beads in 2X SDS loading buffer (100 mM Tris HCl pH 6.8, 4\% SDS, 20\%

518 glycerol, $0.2 \%$ bromophenol blue) and boiling at $95^{\circ} \mathrm{C}$ for 5 minutes. Western blot analyses

519 were performed using monoclonal anti-myc (Cell Signaling, 2276S) at 1:5000 and monoclonal

520 anti-beta actin (abcam, ab8226) at 1:2500 for primary antibodies and HRP-conjugated anti-

521 mouse at 1:5000 for secondary antibodies. Protein A-tagged proteins were detected with HRP-

522 conjugated polyclonal anti-Protein A (Invitrogen, PA1-26853) at 1:5000.

523

$524 \quad$ Telomerase activity assay 
525 The telomerase activity assay was performed as described ${ }^{12}$. Briefly, bead slurries (see

526 immunoprecipitation) were incubated at $30^{\circ} \mathrm{C}$ for 90 minutes in a $10 \mu \mathrm{L}$ reaction containing 50

$527 \mathrm{mM}$ Tris $\mathrm{HCl}$ pH 8.0, 1 mM MgOAc, 5\% glycerol, 1 mM spermidine, $1 \mathrm{mM} \mathrm{DTT}, 100 \mathrm{mM}$ KOAc,

$5280.2 \mathrm{mM}$ dATP, dCTP, and dTTP, $5 \mu \mathrm{M}$ telomerase assay primer (Table S7), and $0.3 \mu \mathrm{M} 3000$

$529 \mathrm{Ci} / \mathrm{mmol}\left[\alpha_{-}{ }^{32} \mathrm{P}\right]$ dGTP (Perkin Elmer, BLU512H250UC). The reaction was stopped with the

530 addition of 0.5\% SDS, $10 \mathrm{mM}$ EDTA pH 8.0, $20 \mathrm{mM}$ Tris $\mathrm{HCl} \mathrm{pH} \mathrm{7.5,} 2 \mu \mathrm{g} / \mu \mathrm{L}$ Proteinase $\mathrm{K}$

531 (Sigma), and $10000 \mathrm{cpm}\left[\gamma_{-}^{-32} \mathrm{P}\right]$ ATP-labeled $100-$ mer oligonucleotide and incubation at $42^{\circ} \mathrm{C}$ for

53215 minutes, followed by phenol: chloroform extraction and ethanol precipitation. RNase A-

533 treated samples were pre-incubated with $20 \mathrm{ng}$ RNase $\mathrm{A}$ (Invitrogen, $\mathrm{AM} 2271$ ) at $30^{\circ} \mathrm{C}$ for 10

534 minutes. Extension products were separated on a 10\% urea-TBE polyacrylamide sequencing gel

535 at $60 \mathrm{~W}$ for 90 minutes, dried and exposed to a PhosphorScreen, and imaged with a Typhoon

536 imager.

538 Genomic DNA extraction, southern blotting, and chromosome fusion PCR

539 Genomic DNA was extracted from logarithmically growing cells according to ${ }^{13}$. For southern

540 blotting, $15 \mu$ genomic DNA was digested overnight with EcoRI and separated on a $1 \%$ agarose

541 gel, transferred to positively charged nylon membranes (Perkin Elmer, NEF988001) by capillary

542 transfer in 10X SSC, and probed with a ${ }^{32} \mathrm{P} \gamma$-ATP-labeled telomere probe (probe sequence

543 provided in Table S7). The chromosome fusion PCR was adapted from ${ }^{12}$. Briefly, $50 \mu L$ PCR

544 reactions contained $1 \mu \mathrm{L}$ genomic DNA, $0.4 \mu \mathrm{M}$ forward and reverse primers (sequences

545 provided in Table S7), $200 \mu \mathrm{M}$ dNTPs, 1X Taq ThermoPol Buffer (NEB), and 1.25 U Taq

546 Polymerase (NEB, M0273). The Trt1 gene was amplified as a loading control in an identical PCR 
547 reaction containing the corresponding primers. The PCR reaction consisted of an initial

548 denaturation at $95^{\circ} \mathrm{C}$ for 5 minutes, followed by 32 cycles of $95^{\circ} \mathrm{C}$ for 15 seconds, $55^{\circ} \mathrm{C}$ for 30

549 seconds, and $68^{\circ} \mathrm{C}$ for 3 minutes, with a final extension at $68^{\circ} \mathrm{C}$ for 10 minutes.

\section{Structure prediction}

552 The structure of S. pombe Bin3 was predicted using Phyre $2^{62}$ and aligned with the co-crystal

553 structure of the $H$. sapiens MePCE methyltransferase domain bound to 7SK snRNA and SAH

$554\left(6 \mathrm{DCB}{ }^{30}\right)$ in PyMol ${ }^{63}$. Primary sequences were aligned with the Clustal Omega Multiple

555 Sequence Alignment tool ${ }^{64}$.

\section{6}

557 Phylogenetic tree construction and search for Bin3-like and Pof8-like sequences

558 Protein sequences (GeneCatalogs) of 472 fungal species were downloaded from the JGI website

559 (https://mycocosm.jgi.doe.gov/) in fasta format. 5614520 proteins were downloaded and

560 merged into a single multifasta file. A blast (Blastp) against all proteins was performed using

561 diamond blastp ${ }^{65}$ with a minimum e-value of $1 \mathrm{e}-5$ and outfmt 6 (for tabular output). In order to

562 identify homologous proteins, proteins were clustered together if they shared at least $50 \%$

563 similarity over $70 \%$ of coverage using SiLiX ${ }^{66}$. When a cluster contained two or more paralogous

564 genes derived from duplications, the species was removed from the cluster. Finally, only

565 clusters with a minimum of 100 species were retained for further analysis. Following these

566 cleaning steps, we obtained 5328 clusters containing one gene per species. Proteins sequences

567 of each cluster were aligned using MAFFT (v7.271) with default parameters

568 (https://mafft.cbrc.jp/alignment/server/). Multiple alignments were cleaned using trimA1 
569 (v1.4.rev22 build [2015-05-21]) with -st 0.01 option. Fastree (version 2.1.10 SSE3) with default

570 parameters were used to build phylogenetic trees ${ }^{67}$. The consensus fungi tree was obtained

571 using ASTRAL (astral.5.6.3) software with default parameters ${ }^{68}$. To detect the presence of

572 genes coding for Bin3-like and Pof8-like proteins, blast searches (blastp and tbalstn) were

573 performed on each studied fungus genome, using initially either the full-length S. pombe Bin3

574 protein sequence or the S. pombe Pof8 xRRM conserved motif. This procedure was repeated in

575 each fungal clade using clade-specific Bin3-like and Pof8-like sequences detected by the initial

576 search using S. pombe sequences. Finally, new blast searches were performed all over again,

577 this time using Bin3-like or Pof8-like "probes" made of a collection of sequences representing

578 the diversity of these two proteins in fungi.

579

580

$\underline{\text { Data availability }}$

581 Mass spectrometry proteomics and RNA Seq data will be supplied prior to publication.

582

583

584

585

586

587

588

589

590 


\section{References:}

592 1. Collins, K. The biogenesis and regulation of telomerase holoenzymes. Nature Reviews Molecular Cell Biology (2006) doi:10.1038/nrm1961.

594 2. Blackburn, E. H. \& Collins, K. Telomerase: An RNP enzyme synthesizes DNA. Cold Spring Harb. Perspect. Biol. (2011) doi:10.1101/cshperspect.a003558.

3. Leonardi, J., Box, J. A., Bunch, J. T. \& Baumann, P. TER1, the RNA subunit of fission yeast telomerase. Nat. Struct. Mol. Biol. (2008) doi:10.1038/nsmb1343.

4. Webb, C. J. \& Zakian, V. A. Identification and characterization of the doi:10.1038/nsmb1354.

5. Greider, C. W. \& Blackburn, E. H. A telomeric sequence in the RNA of Tetrahymena telomerase required for telomere repeat synthesis. Nature (1989) doi:10.1038/337331a0.

6. Feng, J. et al. The RNA component of human telomerase. Science (80-. ). (1995) doi:10.1126/science.7544491.

7. Chapon, C., Cech, T. R. \& Zaug, A. J. Polyadenylation of telomerase RNA in budding yeast. RNA (1997).

8. Box, J. A., Bunch, J. T., Tang, W. \& Baumann, P. Spliceosomal cleavage generates the 3' end of telomerase RNA. Nature (2008) doi:10.1038/nature07584.

$6109 . \quad$ Tang, W., Kannan, R., Blanchette, M. \& Baumann, P. Telomerase RNA biogenesis involves sequential binding by Sm and Lsm complexes. Nature (2012) doi:10.1038/nature10924.

612 10. Witkin, K. L. \& Collins, K. Holoenzyme proteins required for the physiological assembly 
and activity of telomerase. Genes Dev. (2004) doi:10.1101/gad.1201704.

614 11. O'Connor, C. M. \& Collins, K. A Novel RNA Binding Domain in Tetrahymena Telomerase p65 Initiates Hierarchical Assembly of Telomerase Holoenzyme. Mol. Cell. Biol. (2006) doi:10.1128/mcb.26.6.2029-2036.2006.

617 12. Páez-Moscoso, D. J. et al. Pof8 is a La-related protein and a constitutive component of telomerase in fission yeast. Nat. Commun. (2018) doi:10.1038/s41467-017-02284-8.

619 13. Mennie, A. K., Moser, B. A. \& Nakamura, T. M. LARP7-like protein Pof8 regulates telomerase assembly and poly(A)+TERRA expression in fission yeast. Nat. Commun. (2018) doi:10.1038/s41467-018-02874-0.

622 14. Collopy, L. C. et al. LARP7 family proteins have conserved function in telomerase assembly. Nat. Commun. (2018) doi:10.1038/s41467-017-02296-4.

624 15. Hu, X. et al. Quality-Control Mechanism for Telomerase RNA Folding in the Cell. Cell Rep. (2020) doi:10.1016/j.celrep.2020.108568.

626 16. Basu, R., Eichhorn, C. D., Cheng, R., Peterson, R. D. \& Feigon, J. Structure of S. pombe telomerase protein Pof8 C-terminal domain is an xRRM conserved among LARP7 proteins. RNA Biol. (2020) doi:10.1080/15476286.2020.1836891.

629 17. Deragon, J.-M. M. Distribution, organization an evolutionary history of La and LARPs in eukaryotes. RNA Biol. 1-9 (2020) doi:10.1080/15476286.2020.1739930.

631 18. Nguyen, V. T., Kiss, T., Michels, A. A. \& Bensaude, O. 7SK small nuclear RNA binds to and inhibits the activity of CDK9/cyclin T complexes. Nature (2001) doi:10.1038/35104581.

633 19. Yang, Z., Zhu, Q., Luo, K. \& Zhou, Q. The 7SK small nuclear RNA inhibits the CDK9/cyclin T1 kinase to control transcription. Nature (2001) doi:10.1038/35104575. 
635 20. Krueger, B. J. et al. LARP7 is a stable component of the 7SK snRNP while P-TEFb, HEXIM1 and hnRNP A1 are reversibly associated. Nucleic Acids Res. (2008) doi:10.1093/nar/gkn061.

21. Muniz, L., Egloff, S. \& Kiss, T. RNA elements directing in vivo assembly of the 7SK/MePCE/Larp7 transcriptional regulatory snRNP. Nucleic Acids Res. (2013) doi:10.1093/nar/gkt159.

641 22. Eichhorn, C. D., Chug, R. \& Feigon, J. hLARP7 C-terminal domain contains an xRRM that binds the 3' hairpin of 7SK RNA. Nucleic Acids Res. (2016) doi:10.1093/nar/gkw833.

643 23. Jeronimo, C. et al. Systematic Analysis of the Protein Interaction Network for the Human 644 Transcription Machinery Reveals the Identity of the 7SK Capping Enzyme. Mol. Cell (2007) doi:10.1016/j.molcel.2007.06.027.

24. Cosgrove, M. S., Ding, Y., Rennie, W. A., Lane, M. J. \& Hanes, S. D. The bin3 RNA methyltransferase targets 7SK RNA to control transcription and translation. Wiley Interdisciplinary Reviews: RNA (2012) doi:10.1080/09540121.2017.1344767. regulates MicroRNA processing. Cell (2012) doi:10.1016/j.cell.2012.08.041.

651 26. Martinez, A. et al. Human BCDIN3D monomethylates cytoplasmic histidine transfer RNA. Nucleic Acids Res. (2017) doi:10.1093/nar/gkx051.

653 27. Kim, D. U. et al. Analysis of a genome-wide set of gene deletions in the fission yeast Schizosaccharomyces pombe. Nat. Biotechnol. (2010) doi:10.1038/nbt.1628.

655 28. Hayles, J. et al. A genome-wide resource of cell cycle and cell shape genes of fission yeast. Open Biol. (2013) doi:10.1098/rsob.130053. 
657 29. Marz, M. et al. Evolution of 7SK RNA and its protein partners in metazoa. Mol. Biol. Evol. (2009) doi:10.1093/molbev/msp198.

659 30. Yang, Y., Eichhorn, C. D., Wang, Y., Cascio, D. \& Feigon, J. Structural basis of 7SK RNA 5'- $\nu^{-}$ phosphate methylation and retention by MePCE. Nat. Chem. Biol. (2019) doi:10.1038/s41589-018-0188-z.

662 31. Tani, T. \& Ohshima, Y. The gene for the U6 small nuclear RNA in fission yeast has an intron. Nature (1989) doi:10.1038/337087a0.

32. Tani, T. \& Ohshima, Y. mRNA-type introns in U6 small nuclear RNA genes: Implications for the catalysis in pre-mRNA splicing. Genes Dev. (1991) doi:10.1101/gad.5.6.1022.

33. Xue, Y., Yang, Z., Chen, R. \& Zhou, Q. A capping-independent function of MePCE in stabilizing 7SK snRNA and facilitating the assembly of 7SK snRNP. Nucleic Acids Res.

669 34. Gu, J., Patton, J. R., Shimba, S. \& Reddy, R. Localization of modified nucleotides in Schizosaccharomyces pombe spliceosomal small nuclear RNAs: Modified nucleotides are clustered in functionally important regions. RNA (1996).

672 35. Nakamura, T. M. et al. Telomerase catalytic subunit homologs from fission yeast and human. Science (80-. ). (1997) doi:10.1126/science.277.5328.955.

674 36. Beernink, H. T. H., Miller, K., Deshpande, A., Bucher, P. \& Cooper, J. P. Telomere maintenance in fission yeast requires an est1 ortholog. Curr. Biol. (2003) doi:10.1016/S0960-9822(03)00169-6.

677 37. Webb, C. J. \& Zakian, V. A. Schizosaccharomyces pombe Ccq1 and TER1 bind the 14-3-3like domain of Est1, which promotes and stabilizes telomerase-telomere association. 
Genes Dev. (2012) doi:10.1101/gad.181826.111.

38. Lemieux, B. et al. Active Yeast Telomerase Shares Subunits with Ribonucleoproteins

RNase P and RNase MRP. Cell (2016) doi:10.1016/j.cell.2016.04.018.

39. Garcia, P. D. et al. Stability and nuclear localization of yeast telomerase depend on protein components of RNase P/MRP. Nat. Commun. (2020) doi:10.1038/s41467-02015875-9.

40. Lin, K. W. et al. Proteomics of yeast telomerase identified Cdc48-Npl4-Ufd1 and Ufd4 as regulators of Est1 and telomere length. Nat. Commun. (2015) doi:10.1038/ncomms9290.

687 41. Zappulla, D. C. \& Cech, T. R. Yeast telomerase RNA: A flexible scaffold for protein subunits. Proc. Natl. Acad. Sci. U. S. A. (2004) doi:10.1073/pnas.0403641101.

42. Barboric, M. et al. 7SK snRNP/P-TEFb couples transcription elongation with alternative splicing and is essential for vertebrate development. Proc. Natl. Acad. Sci. U. S. A. (2009) doi:10.1073/pnas.0903188106.

692 43. Hasler, D. et al. The Alazami Syndrome-Associated Protein LARP7 Guides U6 Small Nuclear RNA Modification and Contributes to Splicing Robustness. Mol. Cell (2020) doi:10.1016/j.molcel.2020.01.001.

44. Wang, X. et al. LARP7-Mediated U6 snRNA Modification Ensures Splicing Fidelity and Spermatogenesis in Mice. Mol. Cell (2020) doi:10.1016/j.molcel.2020.01.002. the Human Telomerase RNA 3' End. Mol. Cell. Biol. (1999) doi:10.1128/mcb.19.1.567. 
doi:10.1038/nrm2124.

702 47. Lafontaine, D. L. J., Bousquet-Antonelli, C., Henry, Y., Caizergues-Ferrer, M. \& Tollervey,

D. The box $\mathrm{H}+\mathrm{ACA}$ snoRNAs carry Cbf5p, the putative rRNA pseudouridine synthase.

Genes Dev. (1998) doi:10.1101/gad.12.4.527.

705

48. Tseng, C. K., Wang, H. F., Schroeder, M. R. \& Baumann, P. The H/ACA complex disrupts triplex in hTR precursor to permit processing by RRP6 and PARN. Nat. Commun. (2018) doi:10.1038/s41467-018-07822-6.

708

49. Miyoshi, T., Kanoh, J., Saito, M. \& Ishikawa, F. Fission yeast pot1-Tpp1 protects telomeres and regulates telomere length. Science (80-. ). (2008) doi:10.1126/science.1154819.

50. Shukla, S., Schmidt, J. C., Goldfarb, K. C., Cech, T. R. \& Parker, R. Inhibition of telomerase RNA decay rescues telomerase deficiency caused by dyskerin or PARN defects. Nat. Struct. Mol. Biol. (2016) doi:10.1038/nsmb.3184.

713 51. Holohan, B. et al. Impaired telomere maintenance in Alazami syndrome patients with LARP7 deficiency. BMC Genomics (2016) doi:10.1186/s12864-016-3093-4.

715 52. Singh, M. et al. Structural Basis for Telomerase RNA Recognition and RNP Assembly by 716 the Holoenzyme La Family Protein p65. Mol. Cell (2012) doi:10.1016/j.molcel.2012.05.018.

718 53. Bähler, J. et al. Heterologous modules for efficient and versatile PCR-based gene targeting in Schizosaccharomyces pombe. Yeast (1998) doi:10.1002/(SICI)10970061(199807)14:10<943::AID-YEA292>3.0.CO;2-Y.

721 54. Oeffinger, M. et al. Comprehensive analysis of diverse ribonucleoprotein complexes. Nat. Methods (2007) doi:10.1038/nmeth1101. 
723 55. Vakiloroayaei, A., Shah, N. S., Oeffinger, M. \& Bayfield, M. A. The RNA chaperone La promotes pre-tRNA maturation via indiscriminate binding of both native and misfolded targets. Nucleic Acids Res. 45, 11341-11355 (2017).

56. Porat, J. \& Bayfield, M. A. Use of tRNA-Mediated Suppression to Assess RNA Chaperone Function. in RNA Chaperones: Methods and Protocols (ed. Heise, T.) 107-120 (Springer US, 2020). doi:10.1007/978-1-0716-0231-7_6.

57. Bray, N. L., Pimentel, H., Melsted, P. \& Pachter, L. Near-optimal probabilistic RNA-seq quantification. Nat. Biotechnol. (2016) doi:10.1038/nbt.3519.

731 58. Robinson, J. T. et al. Integrative genomics viewer. Nature Biotechnology (2011) doi:10.1038/nbt.1754.

59. Mukherjee, N., Lager, P. J., Friedersdorf, M. B., Thompson, M. A. \& Keene, J. D. Syst. Biol. (2009) doi:10.1038/msb.2009.44.

60. Cox, J. \& Mann, M. MaxQuant enables high peptide identification rates, individualized p.p.b.-range mass accuracies and proteome-wide protein quantification. Nat. Biotechnol. (2008) doi:10.1038/nbt.1511.

61. Drissi, R., Dubois, M. L., Douziech, M. \& Boisvert, F. M. Quantitative proteomics reveals dynamic interactions of the minichromosome maintenance complex (MCM) in the cellular response to etoposide induced DNA damage. Mol. Cell. Proteomics (2015) doi:10.1074/mcp.M115.048991. 
doi:10.1038/nprot.2015.053.

746

63. DeLano, W. L. The PyMOL Molecular Graphics System, Version 1.8. Schrödinger LLC (2014) doi:10.1038/hr.2014.17.

64. Sievers, F. \& Higgins, D. G. Clustal Omega. Curr. Protoc. Bioinforma. (2014) doi:10.1002/0471250953.bi0313s48.

750

65. Buchfink, B., Xie, C. \& Huson, D. H. Fast and sensitive protein alignment using DIAMOND. Nature Methods (2014) doi:10.1038/nmeth.3176.

66. Miele, V., Penel, S. \& Duret, L. Ultra-fast sequence clustering from similarity networks with SiLiX. BMC Bioinformatics (2011) doi:10.1186/1471-2105-12-116.

67. Price, M. N., Dehal, P. S. \& Arkin, A. P. FastTree 2 - Approximately maximum-likelihood trees for large alignments. PLoS One (2010) doi:10.1371/journal.pone.0009490.

68. Mirarab, S. et al. ASTRAL: Genome-scale coalescent-based species tree estimation. in Bioinformatics (2014). doi:10.1093/bioinformatics/btu462.

69. Perez-Riverol, Y. et al. The PRIDE database and related tools and resources in 2019: doi:10.1093/nar/gky1106. 
774 Figure Legends:

775

776 Figure 1: Bin3 interacts with U6 snRNA and the telomerase RNA TER1

777 A) Scatterplot of normalized and log-transformed Control IP TPM against Bin3 IP TPM. Bin3

778 targets of interest are indicated.

779 B) Northern blot analysis of the mature and intron-containing U6 from total RNA and PrA

780 immunoprecipitates from untagged (wild type, wt) and PrA-tagged Bin3 strains.

781 C) Same as (B), for the telomerase RNA TER1.

782

783 Figure 2: Bin3 interacts with the same TER1 species as well-established components of the

784 telomerase holoenzyme

785 A) The 5' and 3' ends of Bin3-, Trt1-, Pof8-, and Lsm3-associated TER1 were identified by cRACE.

786 The results of 10 independent clones per immunoprecipitation are shown below a schematic of

787 the architecture of TER1. 
B) RNase $\mathrm{H}$ northern blots of RNase $\mathrm{H}$ generated 5' and 3' ends of TER1 immunoprecipitated by various telomerase components. total RNA (C) and Bin3-associated transcripts (D).

Figure 3: Bin3 interacts with components of the mature telomerase holoenzyme

794 A) Gene ontology analysis (biological process) of top 50 Bin3 protein interactors. beta actin as a loading control.

Figure 4: Bin3 is recruited to the active telomerase holoenzyme by Pof8

A) Northern blot of TER1 and U6 in PrA immunoprecipitates for an untagged wild type (wt),

801 PrA-tagged (Bin3 PrA), and PrA-tagged Pof8 knockout strain (Bin3 PrA $\triangle$ Pof8). Bin3-PrA was

802 detected in input and immunoprecipitated samples by western blots probing for PrA (bottom

803 panel). Possible cleavage products are indicated with an asterisk.

804 B) Northern blot of mature and intron-containing U6 in PrA- and myc-tagged

805 immunoprecipitated RNA.

806

C) Telomerase assay of myc- (left) and PrA-tagged (right) proteins. A ${ }^{32}$ P-labeled 100-mer

807 oligonucleotide was used as a loading control. Telomerase extension products were compared

808 to a terminal transferase ladder, with +1 and +6 extension products indicated. 
810 locations are based on models constructed by Hu and colleagues ${ }^{15}$ and Mennie and

811 colleagues $^{13}$.

812

813 Figure 5: Bin3 depletion results in an increase in TER1 levels and telomere length

814 A) qRT-PCR of Bin3 mRNA normalized to his1 mRNA (mean \pm standard error, paired $t$ test *

815 at $p>0.05, * *$ at $p<0.01, * * *$ at $p<0.001$, and $* * * *$ at $p<0.0001)$.

816 B) qRT-PCR and a representative northern blot of TER1 from total cellular RNA (mean \pm

817 standard error, paired $t$ test $*$ at $p>0.05, * *$ at $p<0.01, * * *$ at $p<0.001$, and $* * * *$ at

$818 \mathrm{p}<0.0001)$

819 C) Southern blot comparing telomere length in wild type, pof $8 \Delta$, and bin $3 \Delta$ strains transformed

820 with an empty vector or GST-Bin3 following 2 re-streaks on minimal media with or without 15

$821 \mu \mathrm{M}$ thiamine (B1), as indicated.

822

\section{Figure 6: Phylogenic distribution of Bin3 and Pof8 in fungi}

824 Consensus cladogram describing the phylogenic relationships of 472 species representative of

825 fungi phylum and classes and highlighting (using a color code) the distribution of Bin3 and Pof8

826 in these species. The cladogram is a consensus tree of 5328 distinct protein coding gene trees

827 resulting from a genome-wide, against all, protein comparison (see Materials and Methods).

828 Only posterior probabilities inferior to 1 are shown. The Bin3 and Pof8 distribution is

829 recapitulated in Supplemental Table 8 with corresponding protein sequences. A cartoon

830 presenting structural domains of Pof8 is presented. Pof8 La module and RRM1 are only inferred 
831 from secondary structure predictions and are represented by $L a^{*}$ and $R R M 1^{*}$ in the

832 corresponding cartoon. In four species (colored in orange in the cladogram) only an N-terminal

833 truncated version of Pof8 (Pof8*) that cannot accommodate the La module is present. Full

834 cladogram with species names and statistical supports of the different nodes is presented in

835 Figure S5.

836

837

838

839

840 Supplementary figure captions:

841

842 Supplementary figure 1: Bin3 is the essential S. pombe methyl phosphate capping enzyme

843 (MePCE) homolog

844 A) Clustal alignment of the methyltransferase/SAM-binding domains of S. pombe Bin3, $H$.

845 sapiens MePCE, and D. melanogaster Bin3. SAM-binding residues are indicated in red and

846 nucleotide-binding residues are in blue.

847 B) Phyre2 structure prediction ${ }^{62}$ of S. pombe Bin3 bound to a $5^{\prime}$ terminal guanosine (orange)

848 and SAH (green). Predicted SAM-binding residues are in red and nucleotide-binding residues

849 are in blue.

850 C) Wild type and Bin3 knockout strains transformed with pRep4x GST-Bin3 grown on minimal

851 media (EMM-ura) (left) and counter-selected on minimal media with 5 '-fluoroorotic acid (right). 
852 D) Bin3 overexpression results in a slow growth phenotype that is partially rescued with the

853 addition of thiamine (B1). 10 -fold serial dilutions were plated on EMM-ura and grown at $32^{\circ} \mathrm{C}$.

854

855 Supplementary figure 2: Gaussian mixture modeling of the ratio histogram

856 The posterior probabilities of associated Gaussian components for transcripts with a log of odds

857 greater than 3.17 are represented for the Bin3 IP (green line) and control IP (red line).

858

859

860

861 Supplementary figure 3: Reciprocal co-immunoprecipitations validate an interaction between

862 Bin3 and Pof8

863 The interaction between Bin3 and Pof8 was confirmed by $\alpha$-myc co-immunoprecipitation.

864

865 Supplementary figure 4: Bin3 overexpression fails to rescue pof8 $\Delta$-dependent telomere

866 defects

867 Chromosome fusion PCR (top) from wild type and pof8D strains overexpressing the pRep4x

868 empty vector or pRep4x GST-Bin3. The Trt1 gene was amplified from genomic DNA as a loading

869 control (bottom).

870

871 Supplementary figure 5: Phylogenetic distribution of Bin3 and Pof8 in fungi 
bioRxiv preprint doi: https://doi.org/10.1101/2021.04.08.438850; this version posted April 9, 2021. The copyright holder for this preprint (which was not certified by peer review) is the author/funder, who has granted bioRxiv a license to display the preprint in perpetuity. It is made available under aCC-BY 4.0 International license.

872 Complete consensus cladogram describing the phylogenic relationships of 472 species

873 representative of fungi phylum and classes and highlighting (using a color code) the distribution

874 of Bin3 and Pof8 in these species. Only posterior probabilities inferior to 1 are shown.

875

876

877

878

879

880

881

882 
A

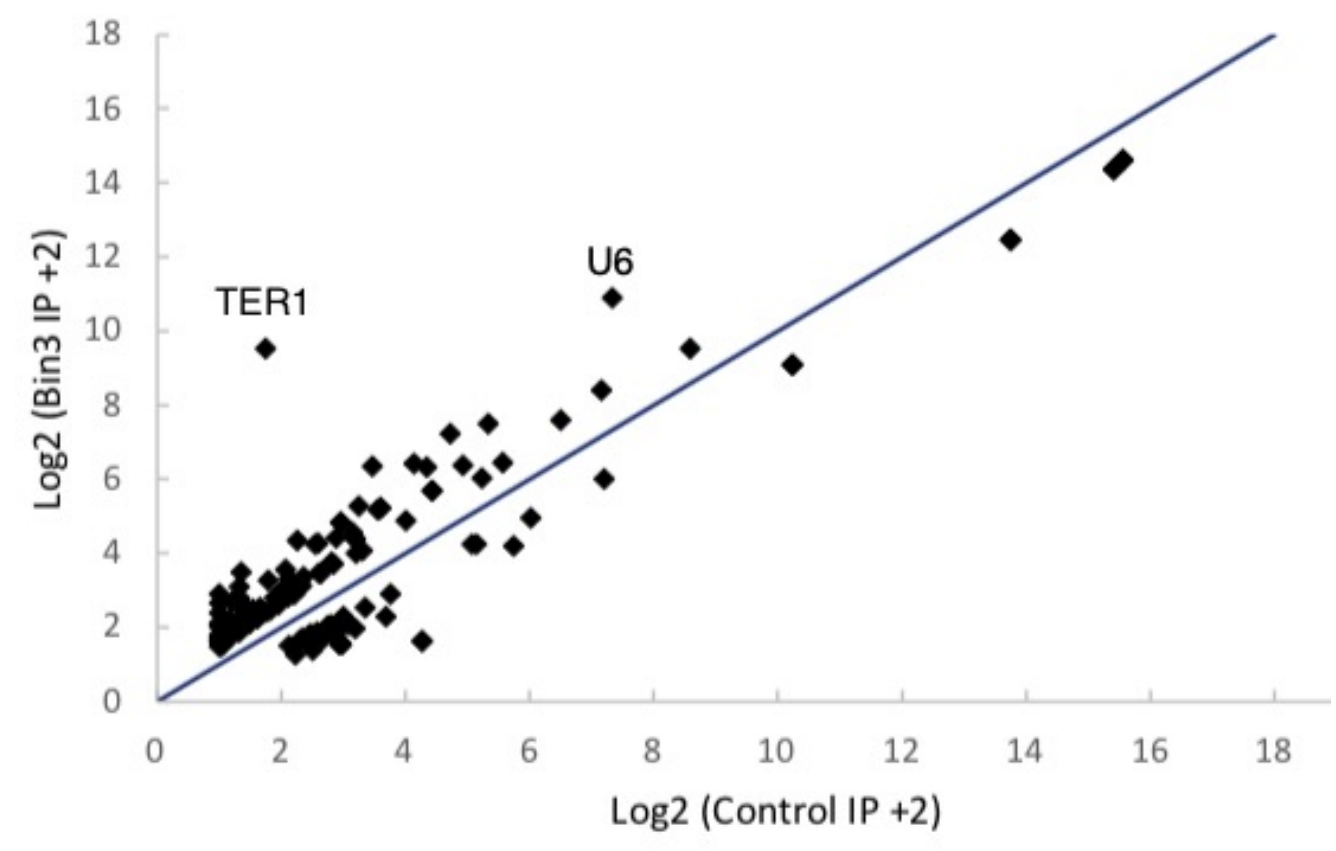

B

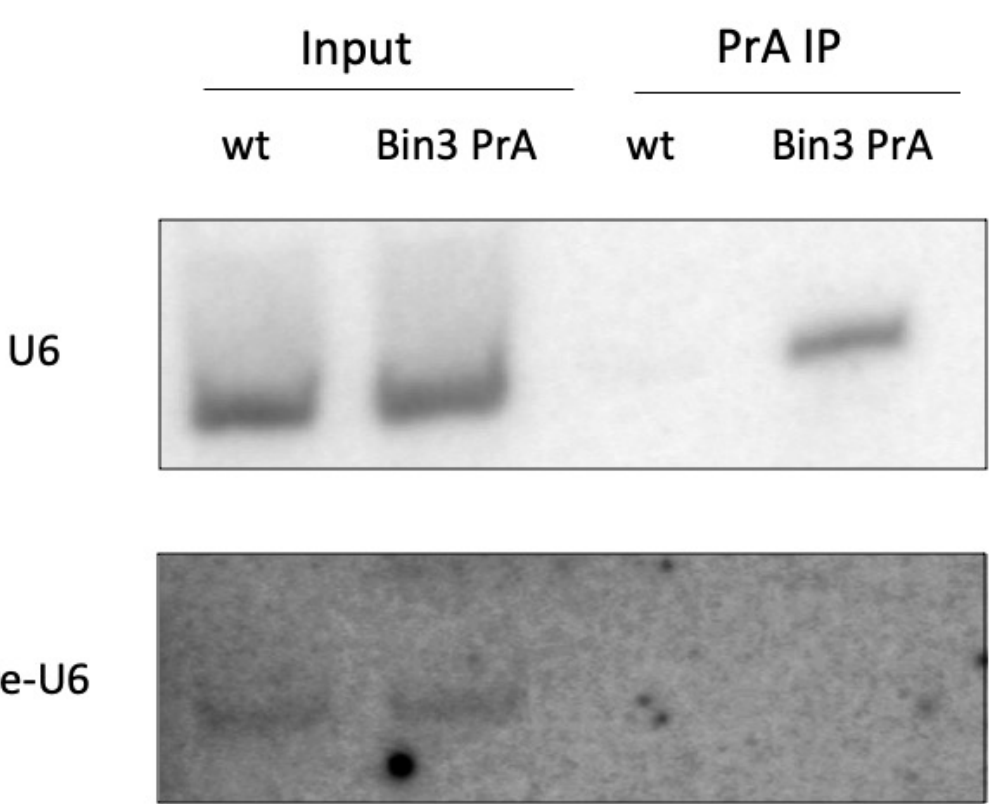

C

Molecular weight

100
90

$-150$

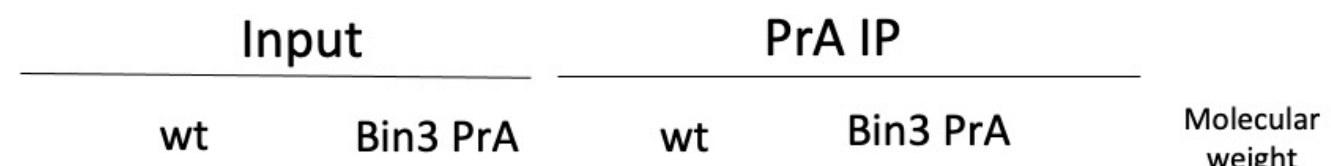

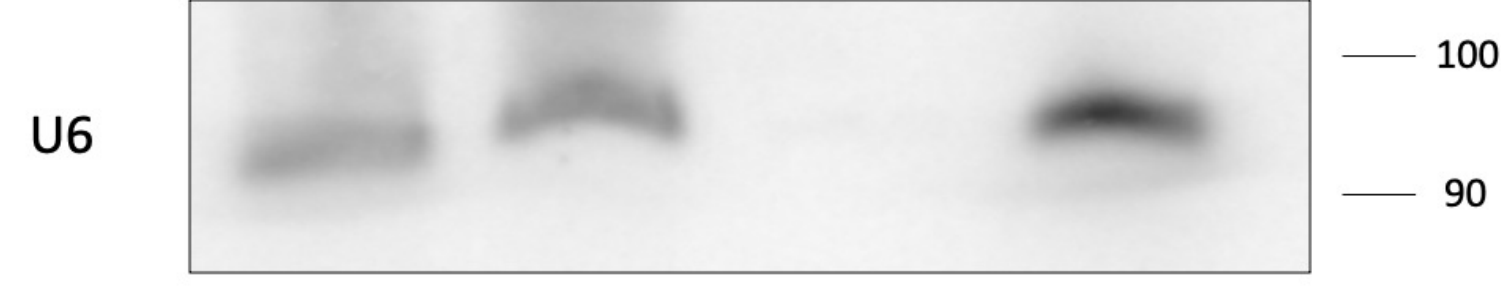

TER1

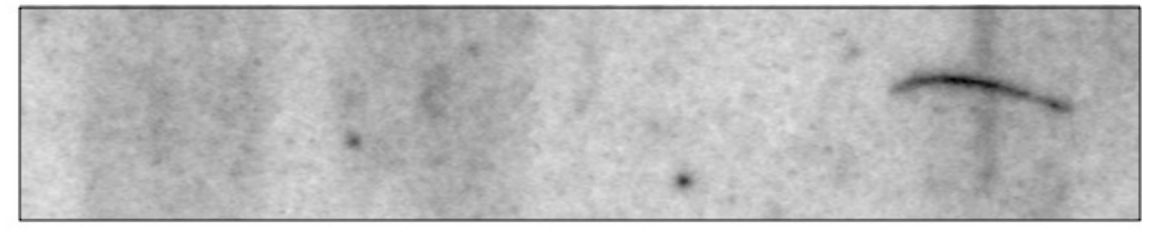

U5

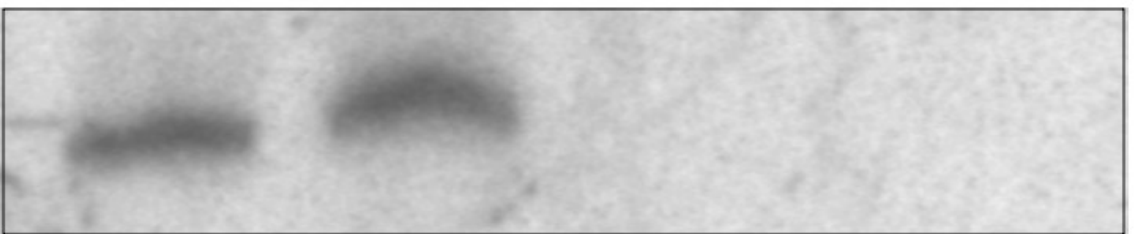


Figure 1: Bin3 interacts with U6 snRNA and the telomerase RNA TER1

A) Scatterplot of normalized and log-transformed Control IP TPM against Bin3 IP TPM. Bin3 targets of interest are indicated.

B) Northern blot analysis of the mature and intron-containing U6 from total RNA and PrA immunoprecipitates from untagged (wild type, wt) and PrA-tagged Bin3 strains.

C) Same as (B), for the telomerase RNA TER1. 
A

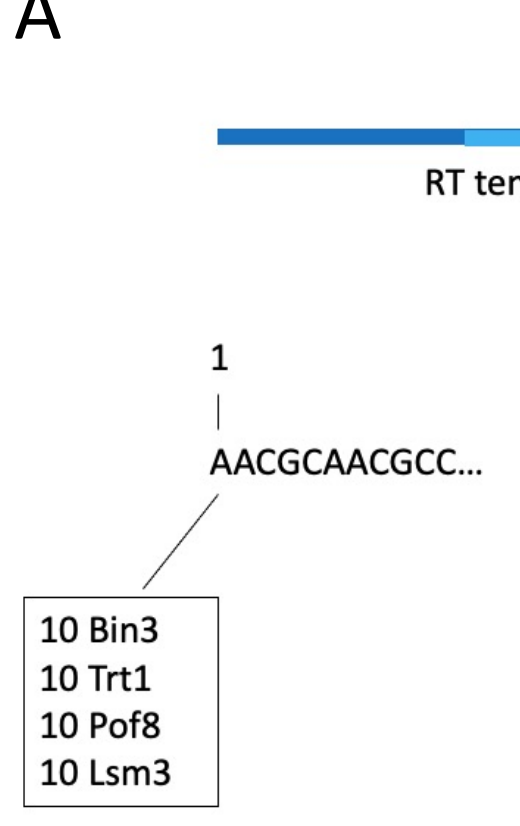

C

$1400 \mathrm{nt}$$$
1200 \mathrm{nt}
$$

Sm binding site

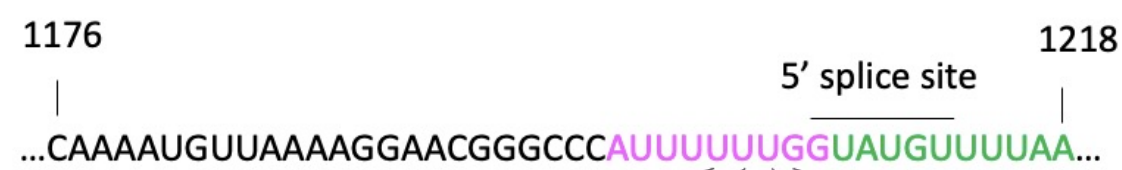

B

$\frac{\text { PrA IP }}{\text { wt } \quad \text { Bin3 PrA }} \frac{\text { Myc IP }}{\text { wt Trt1 myc Pof8 myc Lsm3 myc }} \begin{gathered}\text { Molecular } \\ \text { weight }\end{gathered}$

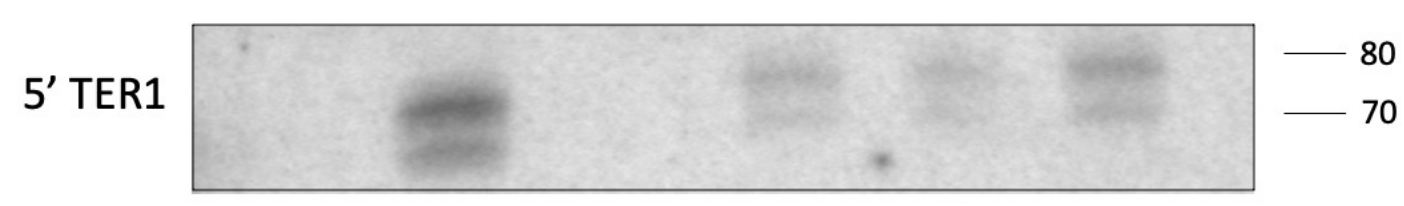

3' TER1

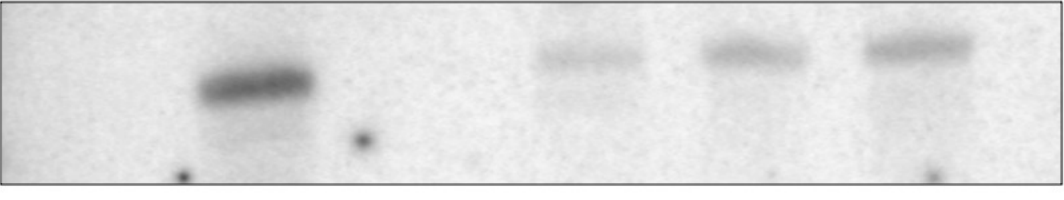

U6

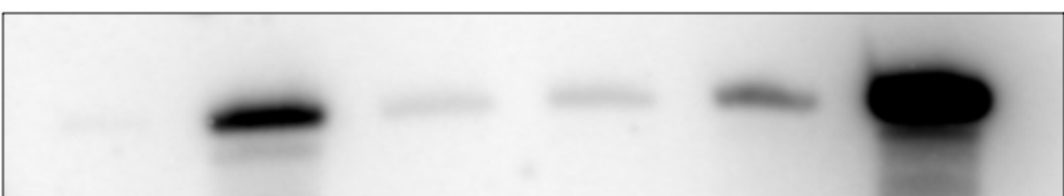

$-100$

$-100$
Total RNA

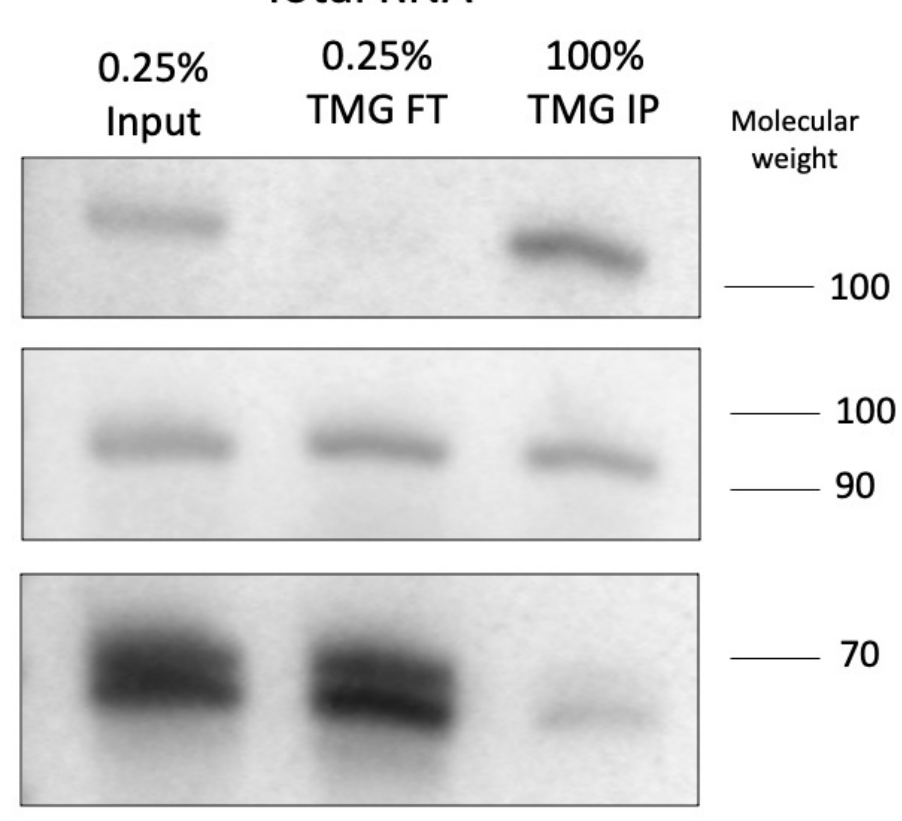

D

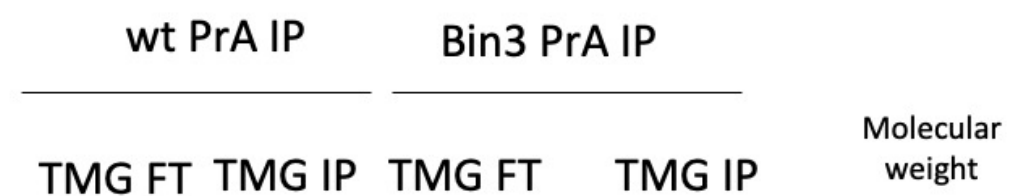

5' TER1

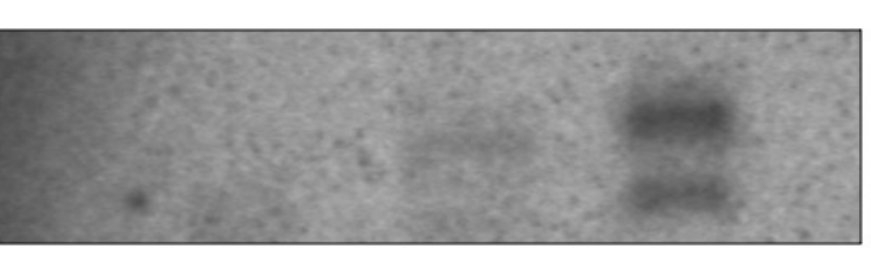

80

U6

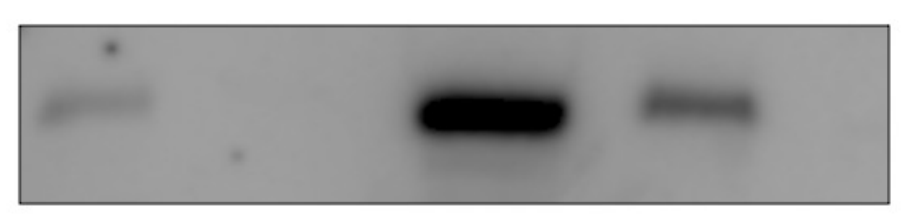

$-100$ 
Figure 2: Bin3 interacts with the same TER1 species as well-established components of the telomerase holoenzyme

A) The 5' and 3' ends of Bin3-, Trt1-, Pof8-, and Lsm3-associated TER1 were identified by cRACE. The results of 10 independent clones per immunoprecipitation are shown below a schematic of the architecture of TER1.

B) RNase H northern blots of RNase H generated 5' and 3' ends of TER1 immunoprecipitated by various telomerase components. C-D) Northern blot of a-TMG flow through (FT) and immunoprecipitated (IP) transcripts from total RNA (C) and Bin3-associated transcripts (D). 
A

\begin{tabular}{|l|}
\hline GO biological process \\
\hline
\end{tabular}

Telomerase holoenzyme complex

Fold enrichment assembly

Telomere maintenance via

31.75

telomerase

RNA-dependent DNA biosynthetic

31.75

process

Ribonucleoprotein complex

5.47

assembly

Ribonucleoprotein complex

subunit organization

5.29

mRNA cis splicing, via

spliceosome

mRNA splicing, via spliceosome

4.78

RNA splicing, via

transesterification reactions with

bulged adenosine as nucleophile

RNA splicing, via

transesterification reactions

RNA splicing
4.72

6.08E-05

1.45E-02

4.78

5.48E-05

1.43E-02

$5.48 \mathrm{E}-05 \quad 1.43 \mathrm{E}-02$

4.52

\section{B}

Input

Flow through

PrA IP

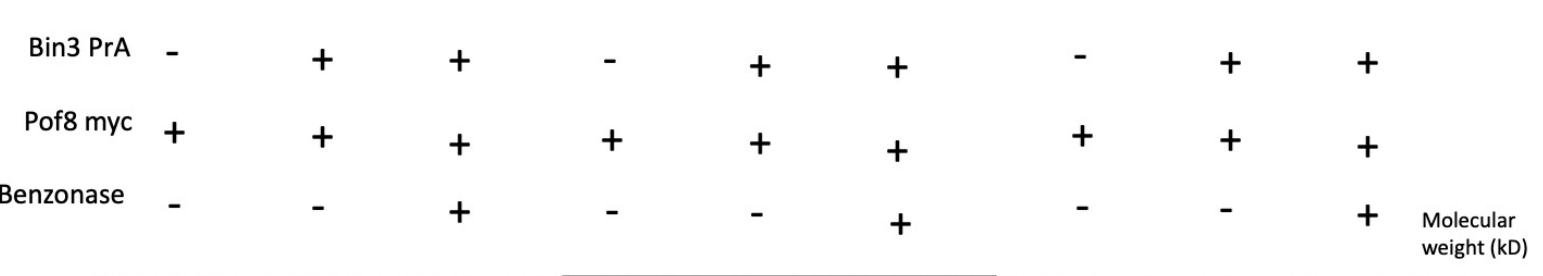

$\boldsymbol{\alpha}$-myc

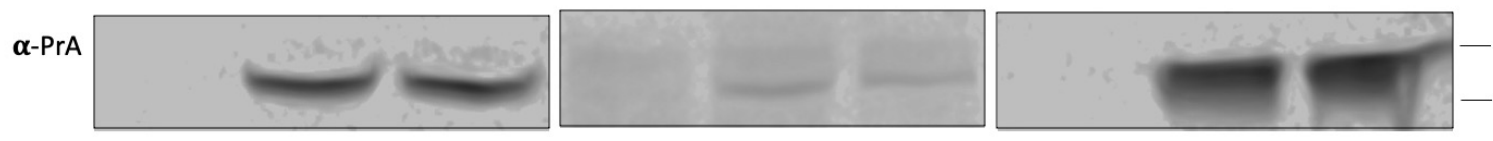

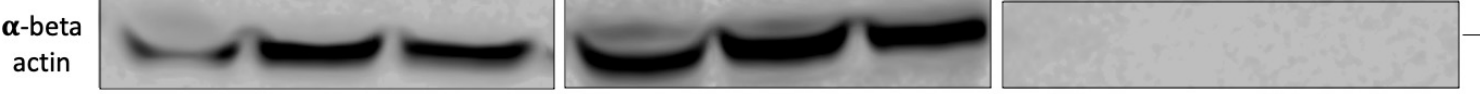

C

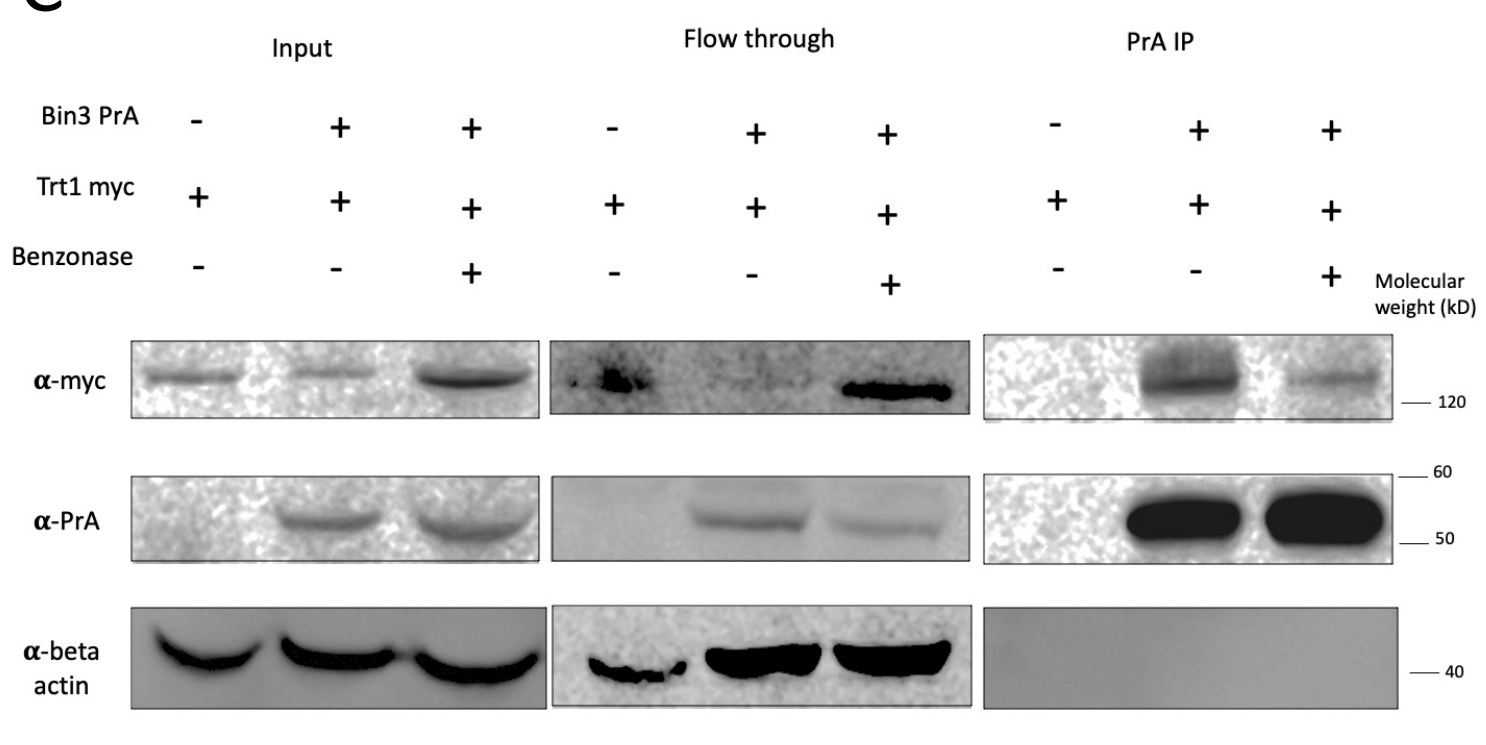


Figure 3: Bin3 interacts with components of the mature telomerase holoenzyme

A) Gene ontology analysis (biological process) of top 50 Bin3 protein interactors.

B-C) Examination and nucleic acid-dependence of interactions between Bin3 and Pof8 (B) and Bin3 and Trt1 (C) by coimmunoprecipitation and western blotting. Blots were reprobed for beta actin as a loading control. 


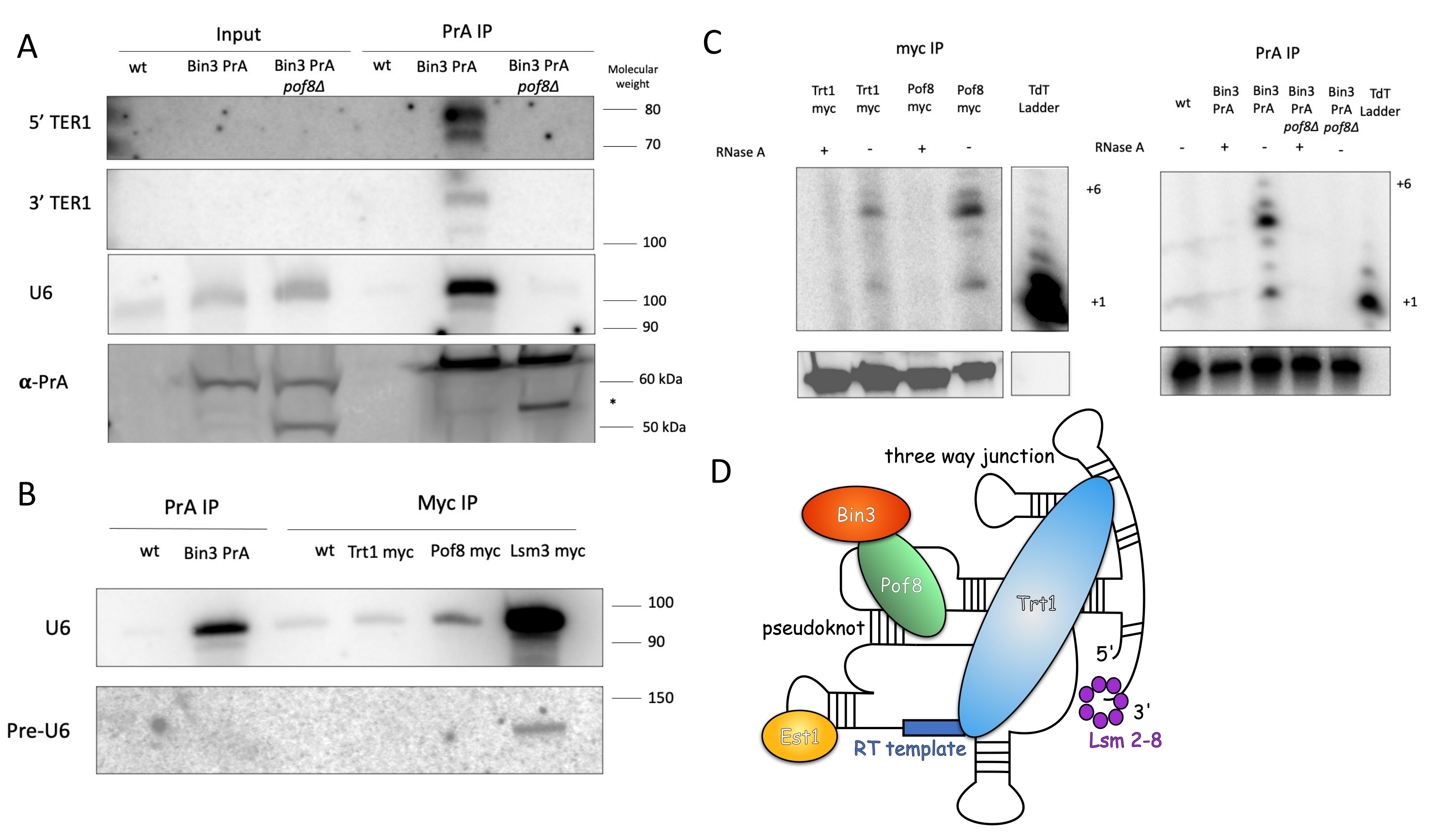




\section{Figure 4: Bin3 is recruited to the active telomerase holoenzyme by Pof8}

A) Northern blot of TER1 and U6 in PrA immunoprecipitates for an untagged wild type (wt), PrA-tagged (Bin3 PrA), and PrA-tagged Pof8 knockout strain (Bin3 PrA pof84). Bin3-PrA was detected in input and immunoprecipitated samples by western blots probing for PrA (bottom panel). Possible cleavage products are indicated with an asterisk.

B) Northern blot of mature and intron-containing U6 in PrA- and myc-tagged immunoprecipitated RNA.

C) Telomerase assay of myc- (left) and PrA-tagged (right) proteins. A ${ }^{32}$ P-labeled 100-mer oligonucleotide was used as a loading control. Telomerase extension products were compared to a terminal transferase ladder, with +1 and +6 extension products indicated.

D) Proposed model of the fission yeast telomerase holoenzyme. TER1 structure and binding locations are based on models constructed by $\mathrm{Hu}$ and colleagues ${ }^{15}$ and Mennie and colleagues ${ }^{13}$. 


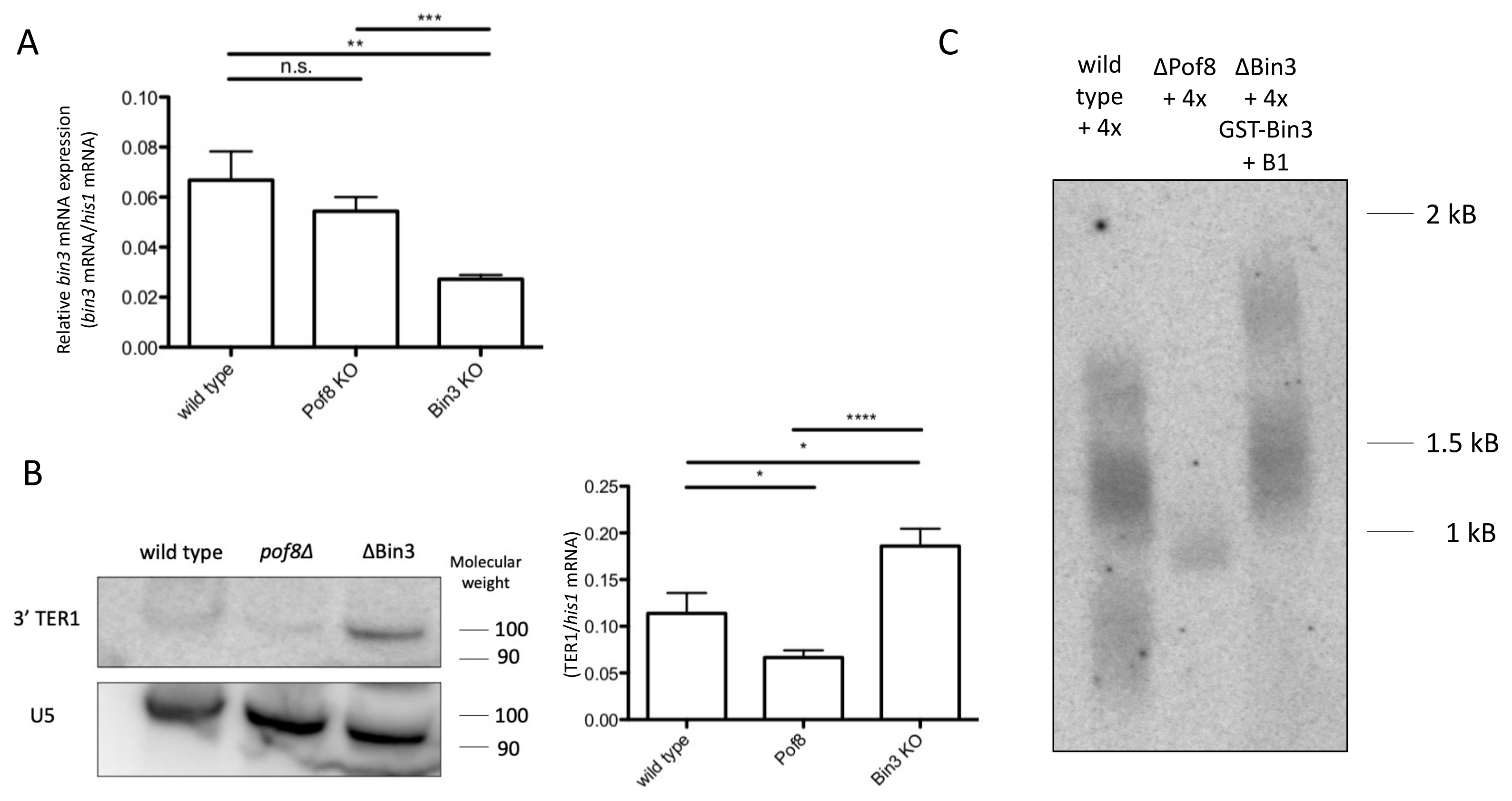


Figure 5: Bin3 depletion results in an increase in TER1 levels and telomere length

A) qRT-PCR of bin3 mRNA normalized to his1 mRNA (mean \pm standard error, paired $t$ test $*$ at $p>0.05, * *$ at $p<0.01, * * *$ at $p<$ paired $t$ test $*$ at $p>0.05, * * 0.001$, and $* * * *$ at $p<0.0001)$.

B) qRT-PCR (normalized to his1 mRNA) and a representative northern blot of TER1 from total cellular RNA (mean \pm standard error, at $p<0.01, * * *$ at $p<0.001$, and $* * * *$ at $p<0.0001)$.

C) Southern blot comparing telomere length in wild type, $\triangle \mathrm{Pof}$, and $\Delta \mathrm{Bin} 3$ strains transformed with an empty vector or GST-Bin3 following 2 re-streaks on minimal media with or without $15 \mu \mathrm{M}$ thiamine (B1), as indicated. 


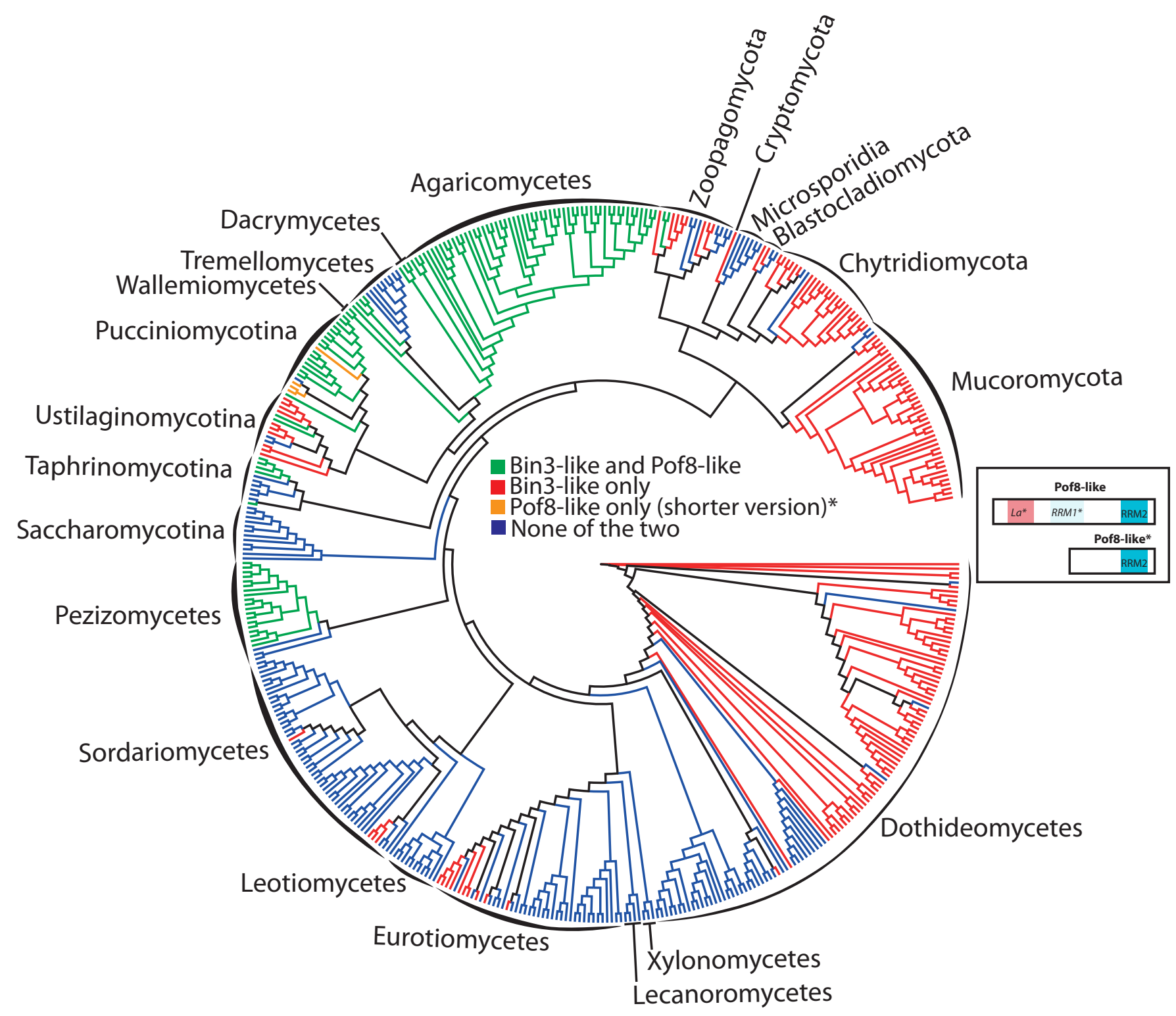




\section{Figure 6: Phylogenic distribution of Bin3 and Pof8 in fungi}

Consensus cladogram describing the phylogenic relationships of 472 species representative of fungi phylum and classes and highlighting (using a color code) the distribution of Bin3 and Pof8 in these species. The cladogram is a consensus tree of 5328 distinct protein coding gene trees resulting from a genome-wide, against all, protein comparison (see Materials and Methods).

The Bin3 and Pof8 distribution is recapitulated in Supplemental Table 8 with corresponding protein sequences. A cartoon presenting structural domains of Pof8 is presented. Pof8 La module and RRM1 are only inferred from secondary structure predictions and are represented by $L a^{*}$ and $R R M 1$ * in the corresponding cartoon. In four species (colored in orange in the cladogram) only an $\mathrm{N}$ terminal truncated version of Pof8 (Pof8*) that cannot accommodate the La module is present. Full cladogram with species names and statistical supports of the different nodes is presented in Figure S5. 


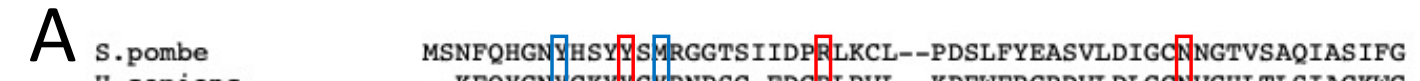
--KFQYGMY CKYY GY RNPSC-EDG GLRVL--KPEWFRGRDVLDLGCAVGHLTLSIACKWG

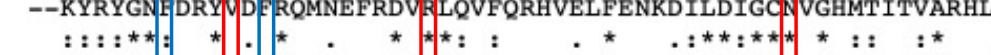
$\because * *: * *$ :

EMM-ura pRep4x-Bin3-GST (ura+) LSEELRLPPQTLEGDPGAEGEE-

S.pombe
H.sapiens D.melanogaster

D.melanogaster

$58 \quad$ C

89

118

118
123
178

178

166
183
238

226
243
298
S.pombe

H.sapiens

S. pombe

D.melanogaste

S. pombe

H.sapiens

D.melanogaster

s.pombe

H.sapiens

D.melanogaster

RIPKEEKLLEVKAEPTVDAKANIAVKDETSGAAH

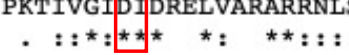

$$
\text { -------------------------------RNPGSIVEDQFNYYPISSIKKFSRIPVQL }
$$
TTR$$
\text { SLLVKPHEFPISFPLTYGRIPR }
$$

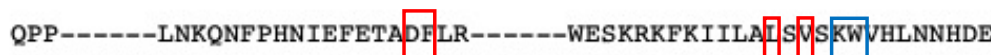
QPP------LNKQNFPNIEFETAPFLR------WESKRKFIILA S S KWHLNNHDE SSSKSPNMLGNKNOFPANVFFRHT NYVLKDESLMASDTQQYDLILCH SUT KW FHLNFGDN

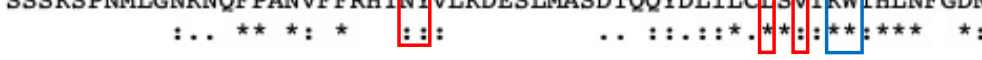

GIIKFFGKISSLLETNGVLILEF Q \&WDS $\mid$ I RAAKKISVFNQTPENLK IQPDAFEHLLNQA GLKRMFRRTYRHLRPGGLVLEE WSS GKR KTLTETIYKNYYRIQLKPEQFSSYLTSP GLKMAFKRMFNQLRPGGKLILEA QWWAS IKKKKNLTPEIYNNYKQIEFFPNKFHEYLLS

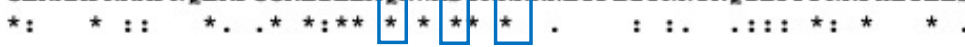

GLVLEYSIEPQVNNSE FR FARKRTMYIYKKKGIGIDVGSSYELVATPHNTSKMFG-RPVYLFHKARSPSH

$: \therefore$. . $\star \star . \star *::: *$

261
278

B

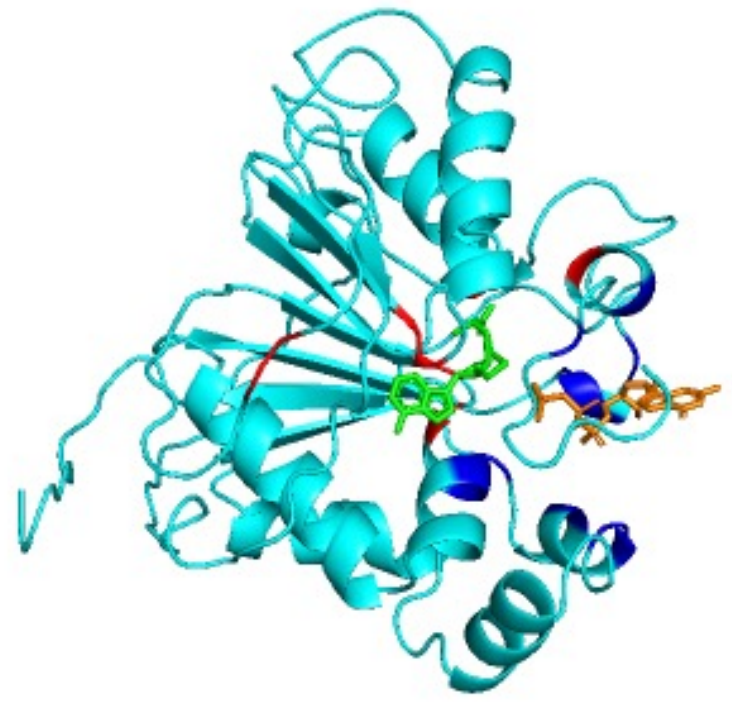

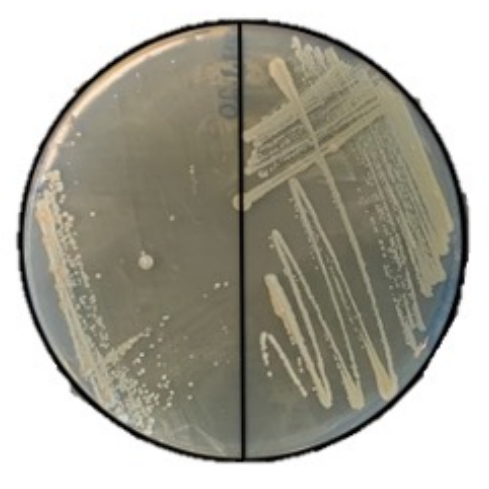
$\triangle$ Bin3 Wild type

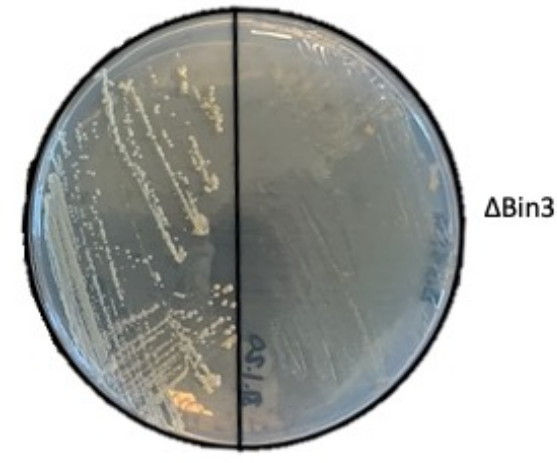

D

(-) B1

(+) $15 \mu \mathrm{M} B 1$

wild type $+4 x$
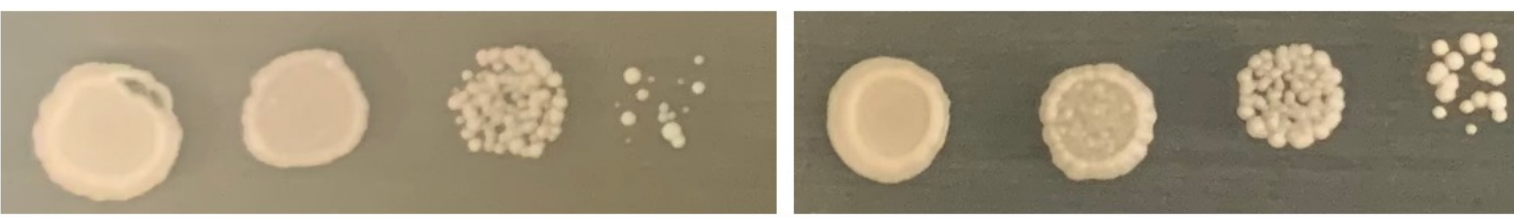

$\Delta \operatorname{Bin} 3+4 x$ GST-Bin 3
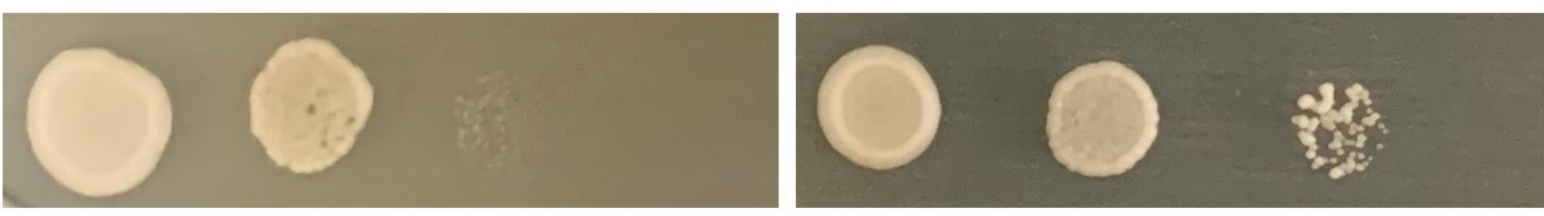


\section{Supplementary figure 1: Bin3 is the essential S. pombe methyl phosphate capping enzyme (MePCE) homolog}

A) Clustal alignment of the methyltransferase/SAM-binding domains of S. pombe Bin3, H. sapiens MePCE, and D. melanogaster Bin3. SAM-binding residues are indicated in red and nucleotide-binding residues are in blue.

B) Phyre 2 structure prediction ${ }^{62}$ of S. pombe Bin3 bound to a $5^{\prime}$ terminal guanosine (orange) and SAH (green). Predicted SAMbinding residues are in red and nucleotide-binding residues are in blue.

C) Wild type and Bin3 knockout strains transformed with pRep4x Bin3 grown on minimal media (EMM-ura) (left) and counterselected on minimal media with 5'-fluoroorotic acid (right).

D) Bin3 overexpression results in a slow growth phenotype that is partially rescued with the addition of thiamine (B1). 10-fold serial dilutions were plated on EMM-ura and grown at $32^{\circ} \mathrm{C}$. 


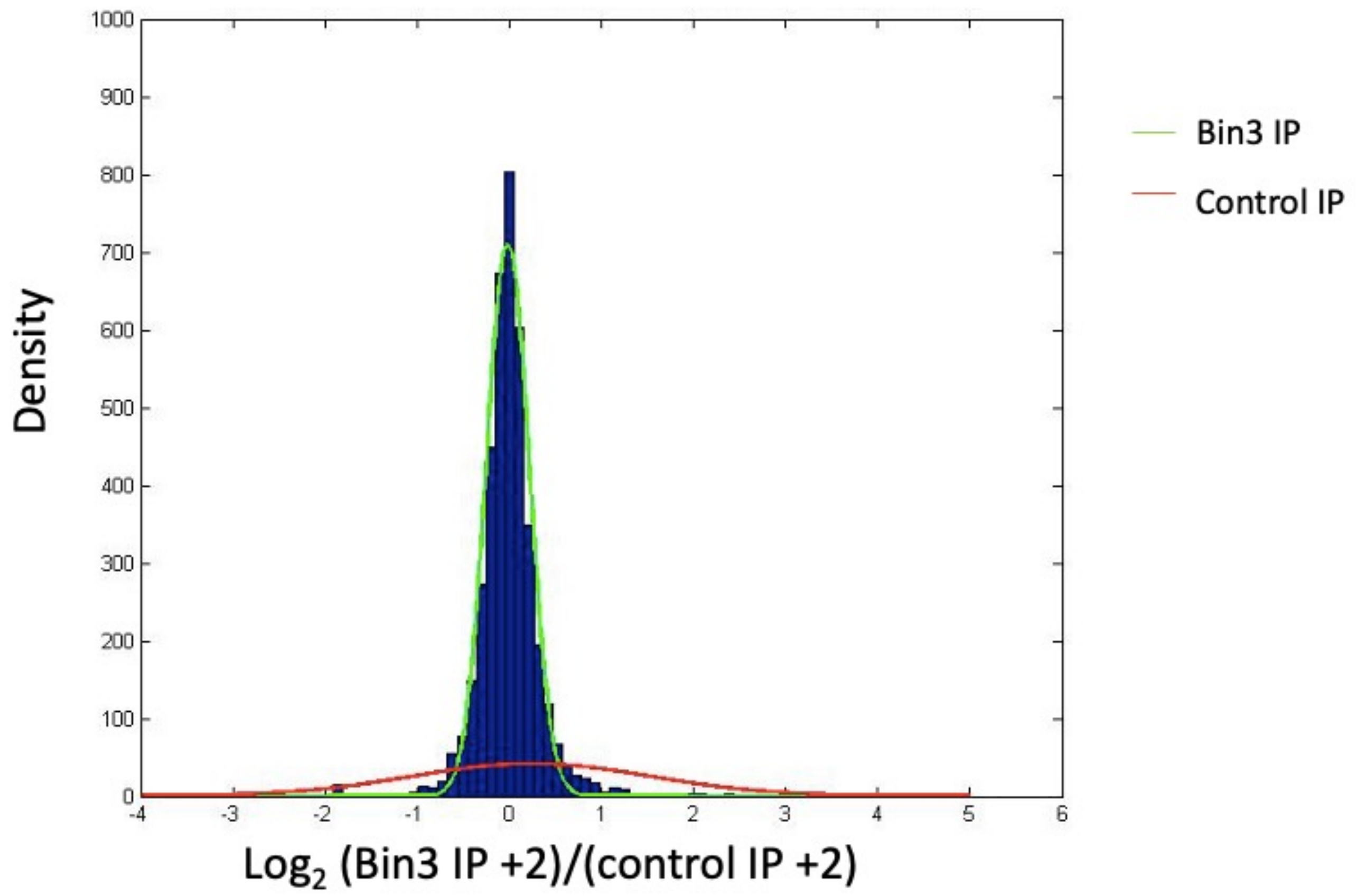




\section{Supplementary figure 2: Gaussian mixture modeling of the ratio histogram}

The posterior probabilities of associated Gaussian components for transcripts with a log of odds greater than 3.17 are represented for the Bin3 IP (green line) and control IP (red line). 


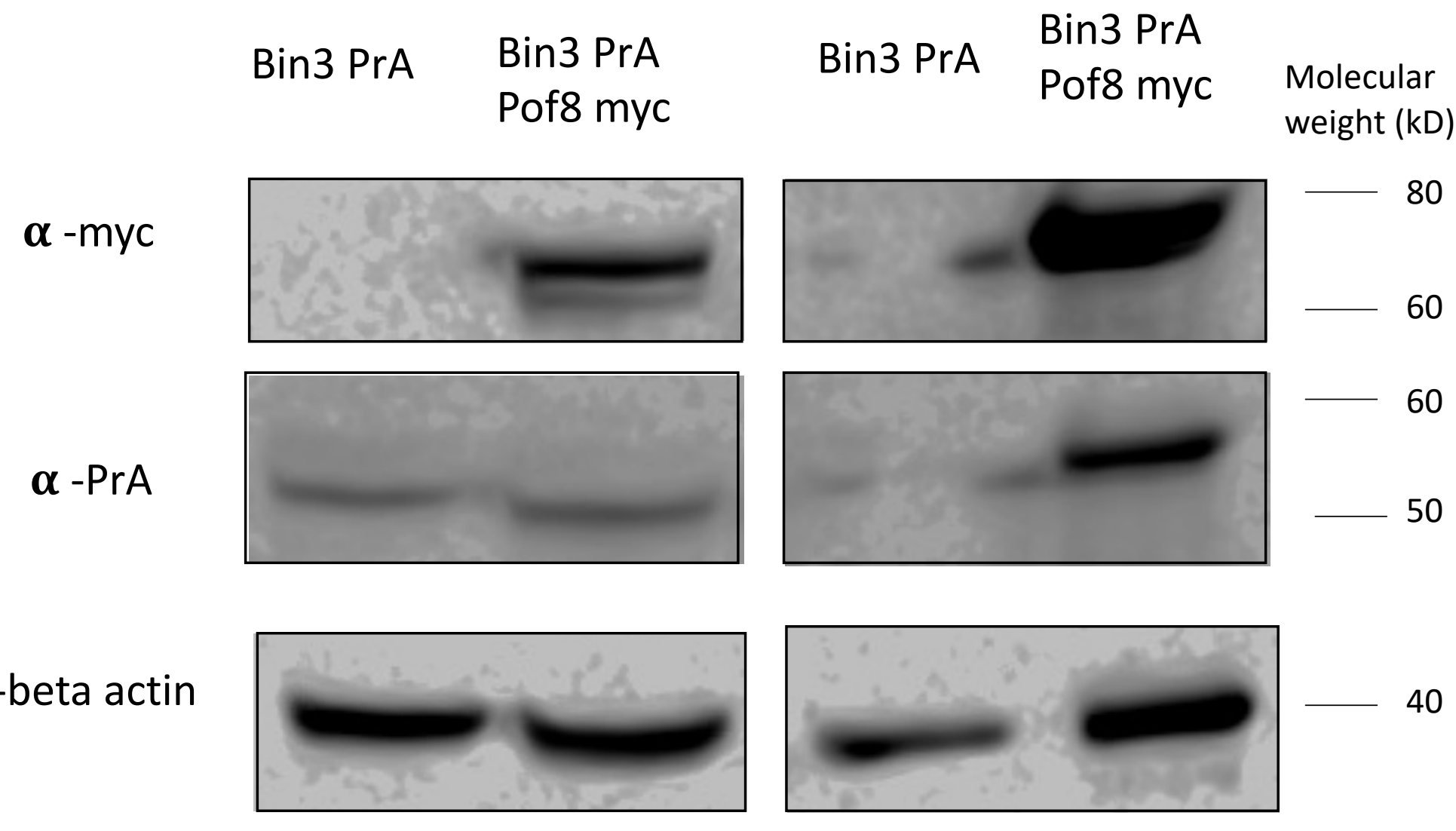


Supplementary figure 3: Reciprocal co-immunoprecipitations validate an interaction between Bin3 and Pof8

The interaction between Bin3 and Pof8 was confirmed by $\alpha$-myc co-immunoprecipitation. 


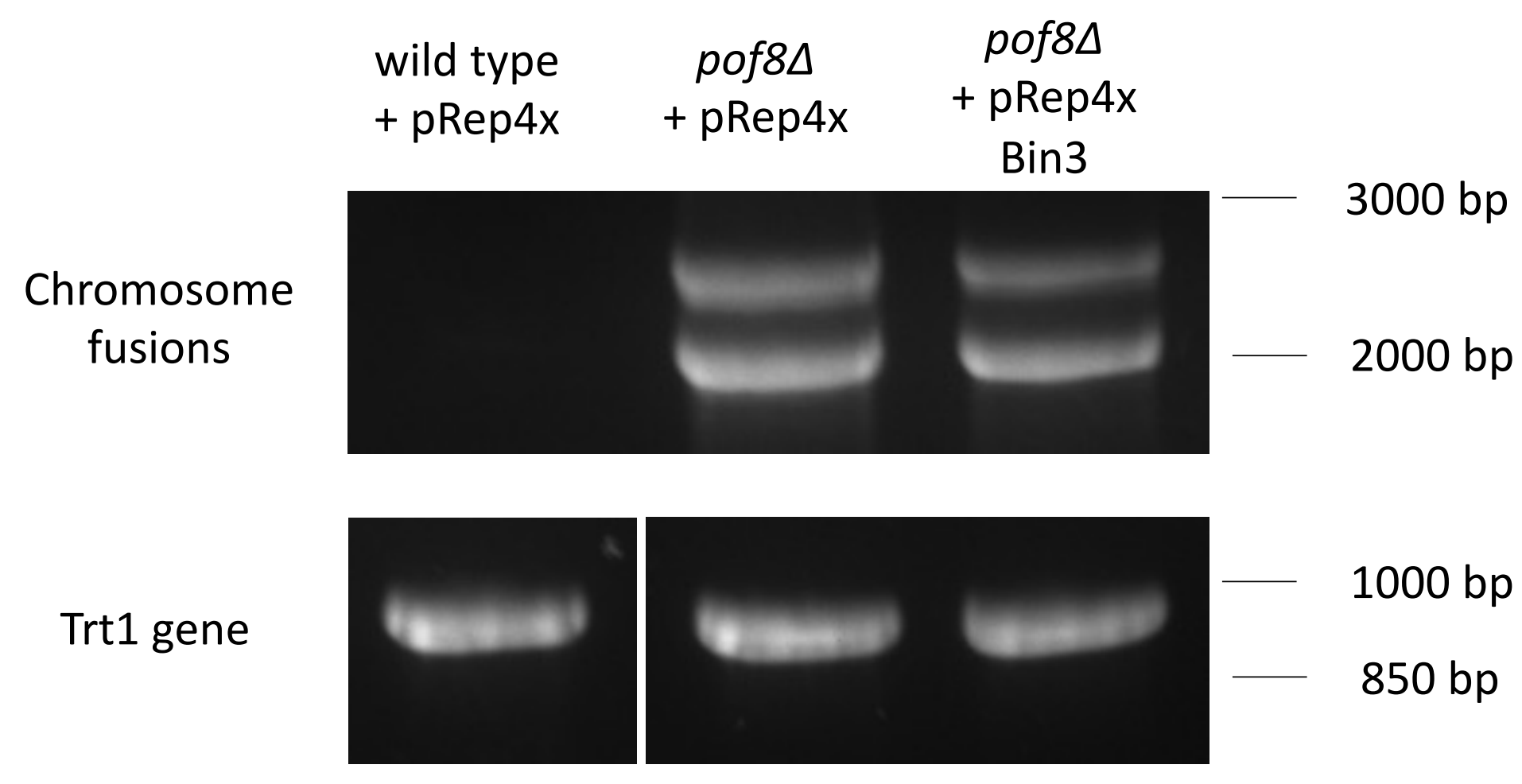


Supplementary figure 4: Bin3 overexpression fails to rescue pof8 $\Delta$-dependent telomere defects

Chromosome fusion PCR (top) from wild type and pof8 $\Delta$ strains overexpressing the pRep4x empty vector or pRep4x Bin3. The Trt1 gene was amplified from genomic DNA as a loading control (bottom). 


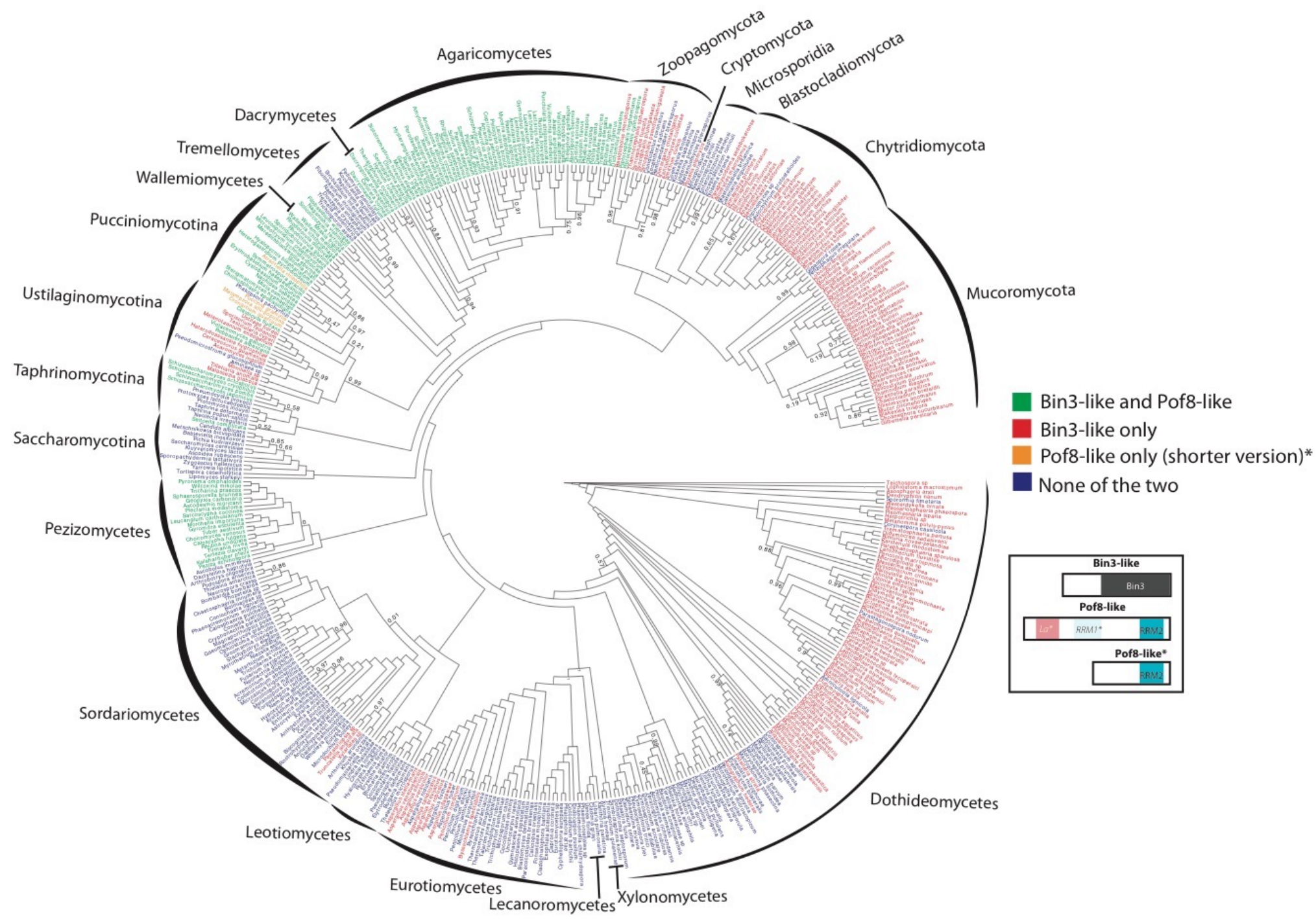


Supplementary figure 5: Phylogenetic distribution of Bin3 and Pof8 in fungi

Complete consensus cladogram describing the phylogenic relationships of 472 species representative of fungi phylum and classes and highlighting (using a color code) the distribution of Bin3 and Pof8 in these species. Only posterior probabilities inferior to 1 are shown. 
List of supplementary tables:

Supplementary table 1: Bin3-associated RNAs were detected by RIP-Seq (attached as spreadsheet)

Transcripts with 4 or more reads in at least one of the replicates, as well as a minimum log of odds value of 3.17, are presented.

Supplementary table 2: Results of Bin3-immunoprecipitated proteins from 2 independent mass spectrometry experiments

Proteins present exclusively in both replicates and absent from control immunoprecipitates were considered. The top 11 interacting proteins are presented, ranked by total peak intensity. Interactions persisting in the presence of benzonase are indicated. Experimentally validated and speculative components of the S. pombe telomerase holoenzyme are shaded.

Supplementary table 3: Top 50 Bin3-associated proteins used for GO analyses.

Supplementary table 4: Gene ontology analysis (cellular component) of the top 50 Bin3 protein interactors

Supplementary table 5: S. pombe strains used in this study

Supplementary Table 6: Primers used to generate tagged strains

Supplementary table 7: probe sequences 
Supplementary Table 8: Distribution of Bin3 and Pof8 in the 472 species used to construct the cladogram of Figure 6 and corresponding protein sequences. (attached as spreadsheet)

Supplementary Table 9: Distribution of Bin3 and Pof8 in basal eukaryotic lineages. Presence

of a Bin3- or LARP7-family member $=Y$, absence $=\mathbf{N}$. 


\section{Supplementary Table 2}

\begin{tabular}{|c|c|c|c|c|}
\hline Protein & Gene & $\begin{array}{l}\text { Bin3 PrA IP } \\
\text { Replicate 1 }\end{array}$ & $\begin{array}{l}\text { Bin3 PrA IP } \\
\text { Replicate } 2\end{array}$ & $\begin{array}{l}\text { Bin3 PrA IP + } \\
\text { benzonase }\end{array}$ \\
\hline LARP7 family protein Pof8 & pof8 & 24 & 24 & 22 \\
\hline $\begin{array}{l}\text { Vigilin KH domain RNA binding } \\
\text { protein }\end{array}$ & $\mathrm{vgl1}$ & 17 & 14 & 20 \\
\hline $\begin{array}{l}\text { Uncharacterized RNA binding } \\
\text { protein }\end{array}$ & SPBC1861 & 16 & 24 & 0 \\
\hline $\begin{array}{l}\text { RNase P and RNase MRP } \\
\text { subunit Pop100 }\end{array}$ & pop100 & 16 & 17 & 0 \\
\hline $\begin{array}{l}\text { Telomerase reverse } \\
\text { transcriptase Trt1 }\end{array}$ & $\operatorname{trt1}$ & 13 & 12 & 0 \\
\hline Bin3 family protein (bait) & SPBC2A9 & 12 & 12 & 11 \\
\hline $\begin{array}{l}\text { WD repeat/ BOP1NT protein } \\
\text { Erb1 }\end{array}$ & erb1 & 9 & 9 & 1 \\
\hline Uncharacterized protein & SPCC18B5 & 7 & 7 & 7 \\
\hline Sec24/Sfb2 subunit & $\sec 24$ & 7 & 3 & 0 \\
\hline Telomerase regulator Est1 & est1 & 6 & 9 & 0 \\
\hline Lsm2-8 complex subunit Lsm6 & Ism6 & 6 & 6 & 0 \\
\hline
\end{tabular}




\section{Supplementary Table 3}

\begin{tabular}{lc}
\hline Protein & Gene \\
LARP7 family protein Pof8 & pof8
\end{tabular}

$\begin{array}{ll}\text { Bin3 family protein (bait) } & \text { SPBC2A9 }\end{array}$

Uncharacterized protein

SPCC18B5

Lsm2-8 complex subunit Lsm8

Ism8

Lsm2-8 complex subunit Lsm7

Ism7

Lsm2-8 complex subunit Lsm2

Ism2

Lsm2-8 complex subunit Lsm6

Ism6

Lsm2-8 complex subunit Lsm5

Ism5

Threonine tRNA ligase

ths1

RNase $\mathrm{P}$ and RNase MRP subunit Pop100

pop100

Periodic tryptophan protein 2 homolog

SPBC713 
Vigilin $\mathrm{KH}$ domain RNA binding protein

Uncharacterized RNA binding protein

Putative glycine tRNA ligase

Small nuclear ribonucleoprotein-associated protein B

Pre-mRNA splicing factor Prp12

Probable mitochondrial import receptor subunit Tom70

Nucleic acid binding protein homolog

Telomerase reverse transcriptase Trt1

Ribosome biogenesis protein Brx1

Ribosome biogenesis protein Erb1

Probable ATP-citrate synthase subunit 2 $\operatorname{vgl1}$

SPBC1861

grs1

smb1

Prp1

tom70

byr3

trt1

brx1

erb1

SPAC22A12 
mRNA export factor Elf1

ATP synthase beta subunit 2

Sm snRNP core protein Smf1

Mitochondrial translation elongation factor EF-Tu

CUE domain-containing protein

Putative G3BP-like protein

Prp19 complex subunit

Vacuolar carboxypeptidase $Y$

RNA binding protein Nop12

Putative mitochondrial ribosome recycling factor

U3 snoRNP-associated protein Utp7 elf1

atp2

smf1

tuf1

SPBC354

$n \times t 3$

prp19

cpy1

nop12

tif451

utp7 
Putative leucine-tRNA ligase Lrs1

Irs1

U5 snRNP complex subunit Spp42

spp42

DNA Polymerase $\mathrm{V}$

pol5

Ribosome assembly factor, WD repeat protein Nsa1

nsa1 
bioRxiv preprint doi: https://doi.org/10.1101/2021.04.08.438850; this version posted April 9, 2021. The copyright holder for this preprint (which was not certified by peer review) is the author/funder, who has granted bioRxiv a license to display the preprint in perpetuity. It is made available under aCC-BY 4.0 International license.

$26 \mathrm{~S}$ protease regulatory subunit 8 homolog

let1 


\section{Supplementary Table 4}

\begin{tabular}{|c|c|c|c|}
\hline GO cellular component & Fold enrichment & Raw P value & False discovery rate \\
\hline Lsm2-8 complex & 42.34 & $8.86 \mathrm{E}-06$ & $1.23 \mathrm{E}-03$ \\
\hline Telomere holoenzyme complex & 41.16 & $6.60 \mathrm{E}-07$ & 2.44E-04 \\
\hline U6 snRNP & 37.04 & $1.32 \mathrm{E}-05$ & $1.63 \mathrm{E}-03$ \\
\hline Spliceosomal tri-snRNP complex & 15.88 & 4.49E-06 & 9.97E-04 \\
\hline U4/U6 x U5 tri-snRNP complex & 15.88 & 4.49E-06 & 8.31E-04 \\
\hline Catalytic step 2 spliceosome & 10.01 & $2.10 \mathrm{E}-04$ & $1.46 \mathrm{E}-02$ \\
\hline Spliceosomal snRNP complex & 8.50 & $2.78 \mathrm{E}-05$ & $2.80 \mathrm{E}-03$ \\
\hline $\begin{array}{l}\text { Small nuclear ribonucleoprotein } \\
\text { complex }\end{array}$ & 8.10 & 3.69E-05 & $3.42 \mathrm{E}-03$ \\
\hline Sm-like protein family complex & 7.98 & $4.05 E-05$ & $3.46 \mathrm{E}-03$ \\
\hline Spliceosomal complex & 6.12 & $7.25 \mathrm{E}-06$ & $1.15 \mathrm{E}-03$ \\
\hline
\end{tabular}




\section{Supplementary Table 5}

\begin{tabular}{|c|c|c|c|c|}
\hline Figure & Strain & Description & Full genotype & Source \\
\hline \multirow{4}{*}{$\begin{array}{l}\text { 1, Table } \\
\text { S1 }\end{array}$} & FY6843 & wild type & $h$ - ura4-D18 & National \\
\hline & & & & BioResource \\
\hline & & & & Project \\
\hline & yJP001 & bin3-PrA & $\begin{array}{l}h \text { - ura4-D18 bin3 }{ }^{+}:: \text {bin3-PrA- } \\
\text { kanMX6 }\end{array}$ & This study \\
\hline \multirow[t]{3}{*}{ S1 } & yAS99 & wild type & h- leu1-32 ura4-D18 ade6-704 & $\begin{array}{l}\text { Intine et al., } \\
2000^{70}\end{array}$ \\
\hline & yJP002 & $\operatorname{bin} 3 \Delta+$ & h- leu1-32 ura4-D18 ade6-704 & This study \\
\hline & & $\begin{array}{l}\text { pRep4x GST- } \\
\text { Bin3 }\end{array}$ & bin3 $\triangle:: n a t M X 6$ pRep4x GST-Bin3 & \\
\hline \multirow[t]{9}{*}{$2 a, b$} & FY6843 & wild type & $h$ - ura4-D18 & National \\
\hline & & & & $\begin{array}{l}\text { BioResource } \\
\text { Project }\end{array}$ \\
\hline & yJP001 & $\operatorname{bin} 3-\operatorname{Pr} A$ & $\begin{array}{l}h \text { - ura4-D18 bin3 }{ }^{+}: \text {bin3-PrA- } \\
\text { kanMX6 }\end{array}$ & This study \\
\hline & TN7706 & trt1-myc & h- leu1-32 ura4-D18 his3-D1 & Mennie et al., \\
\hline & & & $\operatorname{trt1} 1^{+}:: G 8-13 m y c-n a t M \times 6$ & 2018 \\
\hline & AM16932 & pof8-myc & h- leu1-32 ura4-D18 his3-D1 & Mennie et al., \\
\hline & & & pof ${ }^{+}:: 13 m y c-k a n M X 6$ & 2018 \\
\hline & AM17121 & Ism3-myc & h-leu1-32 ura4-D18 his3-D1 & Mennie et al., \\
\hline & & & $\operatorname{Ism} 3^{+}:: 13 m y c-k a n M X 6$ & 2018 \\
\hline \multirow[t]{4}{*}{$2 c$} & FY6843 & wild type & $h$ - ura4-D18 & National \\
\hline & & & & BioResource \\
\hline & & & & Project \\
\hline & yJP001 & bin3-PrA & $\begin{array}{l}\text { h- ura4-D18 bin3 }{ }^{+}: \text {bin3-PrA- } \\
\text { kanMX6 }\end{array}$ & This study \\
\hline \multirow{5}{*}{$\begin{array}{l}\text { 3a, Table } \\
\text { S2, Table } \\
\text { S3, Table } \\
\text { S4 }\end{array}$} & FY6843 & wild type & $h-u r a 4-D 18$ & National \\
\hline & & & & BioResource \\
\hline & & & & Project \\
\hline & & & & \\
\hline & yJP001 & bin3-PrA & $\begin{array}{l}h \text { - ura4-D18 bin3 }{ }^{+}:: \text {bin3-PrA- } \\
\text { kanMX6 }\end{array}$ & This study \\
\hline \multirow[t]{5}{*}{$3 b, s 3$} & yJP001 & bin3-PrA & $\begin{array}{l}h \text { - ura4-D18 bin3 }{ }^{+}: \text {:bin3-PrA- } \\
\text { kanMX6 }\end{array}$ & This study \\
\hline & yJP001a & bin3-PrA & $\begin{array}{l}h+\text { ura4-D18 bin3 }{ }^{+}:: \text {bin3-PrA- } \\
\text { kanMX6 }\end{array}$ & This study \\
\hline & yJP001b & bin3-PrA & $\begin{array}{l}h+\text { ura4-D18 bin3 }{ }^{+}:: \text {bin3-PrA- } \\
\text { bleMX6 }\end{array}$ & This study \\
\hline & AM16932 & pof8-myc & h- leu1-32 ura4-D18 his3-D1 & Mennie et al., \\
\hline & & & pof8 ${ }^{+}:: 13 m y c-k a n M X 6$ & 2018 \\
\hline
\end{tabular}




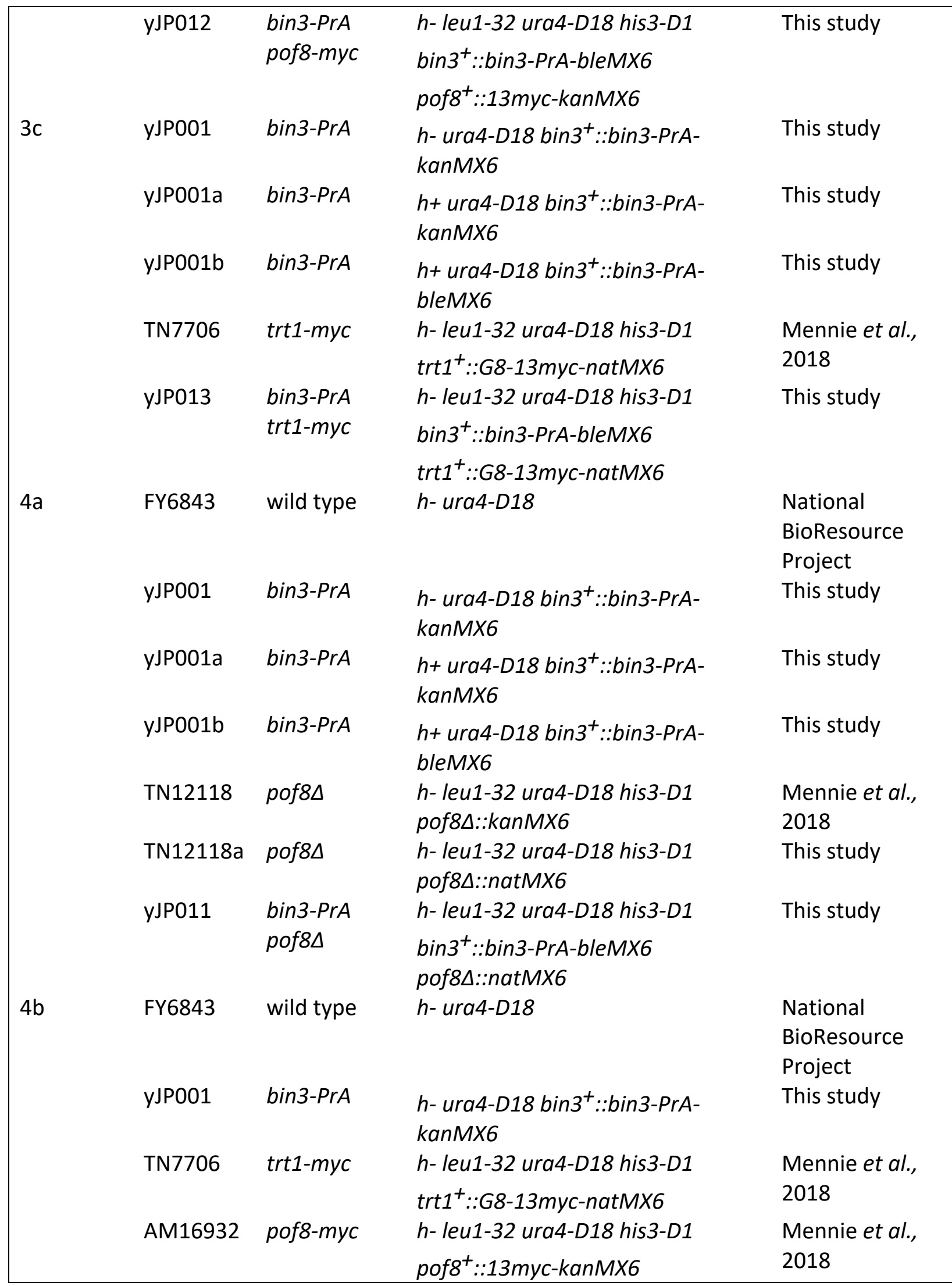




\begin{tabular}{|c|c|c|c|c|}
\hline & AM17121 & Ism3-myc & h- leu1-32 ura4-D18 his3-D1 & Mennie et al., \\
\hline & & & Ism $3^{+}:: 13 m y c-k a n M X 6$ & \\
\hline \multirow[t]{15}{*}{$4 c$} & TN7706 & $\operatorname{trt1}-m y c$ & h-leu1-32 ura4-D18 his3-D1 & Mennie et al., \\
\hline & & & $\operatorname{trt1} 1^{+}:: G 8-13 m y c-n a t M X 6$ & 2018 \\
\hline & AM16932 & pof8-myc & h-leu1-32 ura4-D18 his3-D1 & Mennie et al., \\
\hline & & & pof $8^{+}:: 13 m y c-k a n M X 6$ & 2018 \\
\hline & FY6843 & wild type & $h$ - ura4-D18 & National \\
\hline & & & & BioResource \\
\hline & & & & Project \\
\hline & yJP001 & bin3-PrA & $\begin{array}{l}h \text { - ura4-D18 bin3 }{ }^{+}: \text {bin3-PrA- } \\
\text { kanMX6 }\end{array}$ & This study \\
\hline & yJP001a & bin3-PrA & $\begin{array}{l}h+\text { ura4-D18 bin3 }{ }^{+}:: \text {bin3-PrA- } \\
\text { kanMX6 }\end{array}$ & This study \\
\hline & yJP001b & bin3-PrA & $\begin{array}{l}h+\text { ura4-D18 bin3 }{ }^{+}:: \text {bin3-PrA- } \\
\text { bleMX6 }\end{array}$ & This study \\
\hline & TN12118 & pof8 & $\begin{array}{l}\text { h- leu1-32 ura4-D18 his3-D1 } \\
\text { pof8 } 8:: k a n M \times 6\end{array}$ & $\begin{array}{l}\text { Mennie et al., } \\
2018\end{array}$ \\
\hline & TN12118a & pof8 8 & $\begin{array}{l}\text { h- leu1-32 ura4-D18 his3-D1 } \\
\text { pof8 } 8:: n a t M X 6\end{array}$ & This study \\
\hline & yJP011 & bin3-PrA & h-leu1-32 ura4-D18 his3-D1 & This study \\
\hline & & pof8 8 & bin $3^{+}:$:bin3-PrA-bleMX6 & \\
\hline & & & pof8 $8:: n a t M X 6$ & \\
\hline \multirow[t]{4}{*}{$5 a, 5 b, 5 c$} & yAS99 & wild type & h- leu1-32 ura4-D18 ade6-704 & $\begin{array}{l}\text { Intine et al., } \\
2000\end{array}$ \\
\hline & TN12118 & pof8 & $\begin{array}{l}\text { h- leu1-32 ura4-D18 his3-D1 } \\
\text { pof8 } 8:: k a n M X 6\end{array}$ & $\begin{array}{l}\text { Mennie et al., } \\
2018\end{array}$ \\
\hline & yJP002 & $\operatorname{Bin} \Delta 3+$ & h- leu1-32 ura4-D18 ade6-704 & This study \\
\hline & & $\begin{array}{l}\text { pRep4x GST- } \\
\text { Bin3 }\end{array}$ & bin3 $\triangle:: n a t M X 6$ pRep $4 x$ GST-Bin3 & \\
\hline \multirow[t]{4}{*}{ S4 } & FY6843 & wild type & h-ura4-D18 & National \\
\hline & & & & BioResource \\
\hline & & & & Project \\
\hline & TN12118 & pof8 8 & $\begin{array}{l}\text { h- leu1-32 ura4-D18 his3-D1 } \\
\text { pof8 } 8:: k a n M \times 6\end{array}$ & $\begin{array}{l}\text { Mennie et al., } \\
2018\end{array}$ \\
\hline
\end{tabular}




\section{Supplementary Table 6}

\begin{tabular}{|c|c|}
\hline Primer & Sequence \\
\hline Bin3 PrA & 5'CATTTGCTTAATCAAGCAGGACTAGTGCTTGAATACAGTATCGAACCTCA \\
\hline For (tag & AGTAAATAACTCTGAGTACAAGAATTTTGCCAAACGAACAATGTATAAAAA \\
\hline \multirow[t]{2}{*}{ integration) } & AAAAGGAATTGGAATCATAAAACTATTAACTATTAACTTCTACTCGTACGCT \\
\hline & GCAGGTCGAC 3' \\
\hline Bin3 PrA & 5'CTACTTCATTCCGCTTTTCTTTCTTTCCTCATATGTTGAATTAGAGAATTTAAT \\
\hline Rev (tag & AAAAAATGAGACACGTCCTTTTGGGACATTATGCTTTATAGTTCCAGTCATTGT \\
\hline integration) & CTATTCTTAACGTTTTACTAGACAAATCGATGAATTCGATGAATTCGAGCTCG 3' \\
\hline $\begin{array}{l}\text { MX6 For } \\
\text { (antibiotic } \\
\text { marker } \\
\text { swap) }\end{array}$ & 5' GACATGGAGGCCCAGAATAC 3' \\
\hline $\begin{array}{l}\text { MX6 Rev } \\
\text { (antibiotic } \\
\text { marker } \\
\text { swap) }\end{array}$ & 5' TGGATGCGCGCGTTAGTATC 3' \\
\hline Bin3 KO For & 5'TTCGATTCGTAAGTTTAAATTCCATTGCAAAATTTATACTTTTATAAAAAATA \\
\hline $\begin{array}{l}\text { (Bin3 gene } \\
\text { disruption) }\end{array}$ & GGGGTGATTTGAAAAACCTCCTCGACTTGGGCGGATCCCCGGGTTAATTAA 3' \\
\hline Bin3 KO Rev & 5'GAGAATTTAATAAAAAATGAGACACGTCCTTTTGGGACATTATGCTTTATA \\
\hline (Bin3 gene & GTTCCAGTCATTGTCTATTCTTAACGTTTTAACTAGACAAGAATTCGAGCTCGTT \\
\hline disruption) & TAAAC 3' \\
\hline
\end{tabular}




\section{Supplementary Table 7}

\begin{tabular}{|c|c|c|}
\hline Figure & Probe & Sequence \\
\hline $\begin{array}{l}1 c, 1 d, \\
2 b, 3 c, \\
2 d, 4 a, \\
4 b\end{array}$ & U6 & 5' GTGTCATCCTTGTGCAGGGGCCAT 3' \\
\hline $1 c, 4 b$ & U6 intron & 5' CAACGAGTTAGTATGACTCGAACCTTGG 3' \\
\hline $1 d$ & Ter1 & 5' CCCGTTCCTTTTAACATTTTTGCTCAGACCAAGTG 5' \\
\hline $\begin{array}{l}1 d, 2 c, \\
5 b\end{array}$ & U5 & 5' CTGGTAAAAGGCAAGAAACAGATACG 3' \\
\hline \multirow[t]{2}{*}{$2 a$} & $\begin{array}{l}\text { Ter1 cRACE } \\
\text { For }\end{array}$ & 5’ TCTTGAGCGCGTTTTAGGTTTTTTTTCACTT 3' \\
\hline & $\begin{array}{l}\text { Ter1 cRACE } \\
\text { Rev }\end{array}$ & 5' AACAACGGACGAGCTACACTCAAAATATTA 3' \\
\hline \multirow[t]{4}{*}{$\begin{array}{l}2 b, 4 a \\
4 b, 5 b\end{array}$} & $\begin{array}{l}\text { Ter1 3' } \\
\text { RNase H }\end{array}$ & 5' GGGTAGTCAACTATTTTACTAGCG 3' \\
\hline & $\begin{array}{l}\text { Ter1 5' } \\
\text { RNase H }\end{array}$ & 5' TTTCAAATCAATCACCAAGCCC $3^{\prime}$ \\
\hline & $\begin{array}{l}\text { Ter1 3' } \\
\text { RNase H } \\
\text { northern }\end{array}$ & 5' GCTCAGACCAAGTGAAAAAAACC 3' \\
\hline & $\begin{array}{l}\text { Ter1 5' } \\
\text { RNase H } \\
\text { northern }\end{array}$ & 5' TCCTTGTCAACCTTCTAAGCATG 3' \\
\hline 2c & tRNA Thr ${ }^{\mathrm{CGU}}$ & 5' TGCTCCGGCAGTGACTCGAACA 3' \\
\hline $4 c$ & $\begin{array}{l}\text { Telomerase } \\
\text { assay primer }\end{array}$ & 5' GTTACGGTTACAGGTTACG 3' \\
\hline \multirow[t]{4}{*}{$5 a$} & $\begin{array}{l}\text { Bin3 mRNA } \\
5^{\prime} \mathrm{qPCR}\end{array}$ & 5' GAGGAACCTCAATTATCGATCCAAGACTTAAATGTTTGC 3' \\
\hline & $\begin{array}{l}\text { Bin3 mRNA } \\
3^{\prime} q P C R\end{array}$ & 5' TCGAGCTTTTTTGGATGAGAACATGATCGATATCCAATCC 3' \\
\hline & His1 5' qPCR & 5' TGTCCACCTCGGAATCACTG 3' \\
\hline & His1 3' qPCR & 5' CGAAGACGTGCTTCAGCGA 3' \\
\hline \multirow[t]{2}{*}{$5 b$} & Ter1 5' qPCR & CAGTGTACGTGAGTCTTCTGCCTT \\
\hline & Ter1 3’ qPCR & CAAAAATTCGTTGTGATCTGACAAG \\
\hline $5 c$ & $\begin{array}{l}\text { Telomere } \\
\text { southern } \\
\text { probe }\end{array}$ & 5' GGGTTACATGTTACAGGTTACA 3' \\
\hline \multirow[t]{4}{*}{ S3 } & $\begin{array}{l}\text { Chromosome } \\
\text { fusion For }\end{array}$ & 5' GGGTTGCAAAGTATGATTGTGGTAA 3' \\
\hline & $\begin{array}{l}\text { Chromosome } \\
\text { fusion Rev }\end{array}$ & 5' TGTTGAATGTCAGAACCAACTGTTGCAT 3' \\
\hline & Trt1 For & 5' GCAAAGAAGTTTCCTGGAATAGC 3' \\
\hline & Trt1 Rev & 5' GATGTAATAAAGGGTCGGCAC 3' \\
\hline
\end{tabular}




\section{Supplementary Table 9}

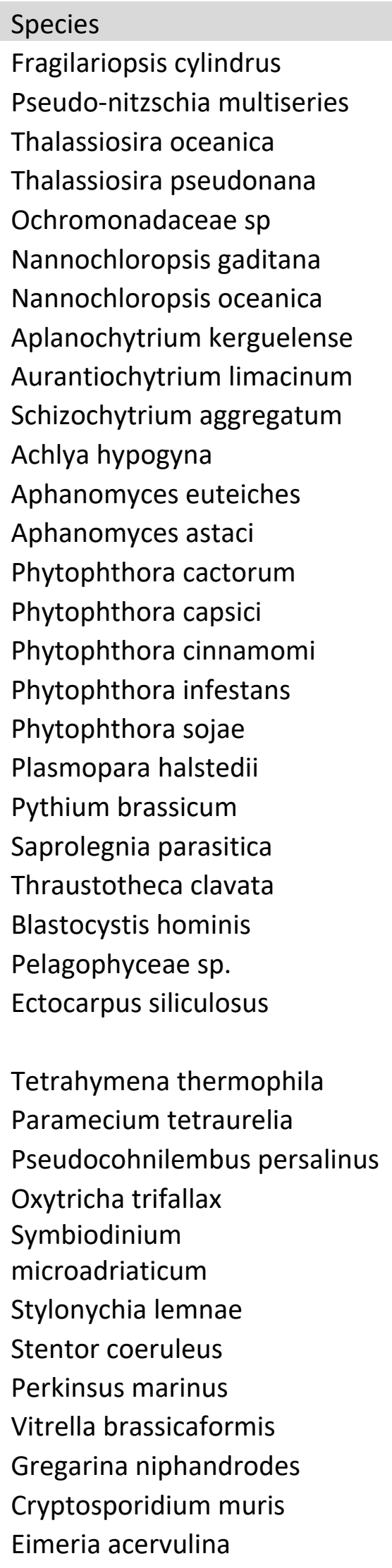

Classification

Stramenopiles, Bacillariophyta

Stramenopiles, Bacillariophyta

Stramenopiles, Bacillariophyta

Stramenopiles, Bacillariophyta

Stramenopiles, Chrysophyta

Stramenopiles, Eustigmatophyceae

Stramenopiles, Eustigmatophyceae

Stramenopiles, Labyrinthulomycetes

Stramenopiles, Labyrinthulomycetes

Stramenopiles, Labyrinthulomycetes

Stramenopiles, Oomycota

Stramenopiles, Oomycota

Stramenopiles, Oomycota

Stramenopiles, Oomycota

Stramenopiles, Oomycota

Stramenopiles, Oomycota

Stramenopiles, Oomycota

Stramenopiles, Oomycota

Stramenopiles, Oomycota

Stramenopiles, Oomycota

Stramenopiles, Oomycota

Stramenopiles, Oomycota

Stramenopiles, Opalozoa

Stramenopiles, Pelagophyceae

Stramenopiles, Phaeophyceae

Alveolates, Ciliophora

Alveolates, Ciliophora

Alveolates, Ciliophora

Alveolates, Ciliophora

Alveolates, Dinophyta

Alveolates, Ciliophora

Alveolates, Ciliophora

Alveolates, Perkinsea

Alveolates, Chromorida

Alveolates, Apicomplexa

Alveolates, Apicomplexa

Alveolates, Apicomplexa
BIN3

LARP7

$\mathrm{N}$

$\mathrm{N}$

$\mathrm{N} \quad \mathrm{N}$

$\mathrm{N} \quad \mathrm{N}$

$\mathrm{N} \quad \mathrm{N}$

Y N

$\begin{array}{ll}Y & N\end{array}$

$\mathrm{Y} \quad \mathrm{N}$

$\mathrm{N} \quad \mathrm{N}$

$\mathrm{N} \quad \mathrm{N}$

$\mathrm{N} \quad \mathrm{N}$

$\begin{array}{ll}\mathrm{Y} & \mathrm{Y}\end{array}$

$Y \quad Y$

$Y \quad Y$

$y \quad y$

$y \quad y$

$y \quad y$

$y \quad y$

$y \quad y$

$y \quad y$

$Y \quad Y$

$\begin{array}{ll}Y & Y\end{array}$

$\begin{array}{ll}Y & Y\end{array}$

$\mathrm{N} \quad \mathrm{N}$

$Y \quad Y$

$Y \quad Y$

$Y \quad Y$

$\begin{array}{ll}Y & Y\end{array}$

Y N

$\begin{array}{ll}Y & Y\end{array}$

$\begin{array}{ll}Y & N\end{array}$

$Y \quad Y$

Y N

$\begin{array}{ll}Y & N\end{array}$

$\begin{array}{ll}\mathrm{Y} & \mathrm{N}\end{array}$

$\mathrm{N} \quad \mathrm{N}$

$\mathrm{N} \quad \mathrm{N}$

Y N 
Toxoplama gondii

Neospora caninum

Cyclospora cayetanensis

Cystoisospora suis

Plasmodiophora brassicae

Trypanosoma brucei

Naegleria gruberi

Naegleria fowleri

Giardia lamblia

Giardia intestinalis

Trichomonas vaginalis

Emiliania huxleyi

Chrysochromulina tobinii

Pavlovalas sp

Phaeocystis globosa
Alveolates, Apicomplexa

Alveolates, Apicomplexa

Alveolates, Apicomplexa

Alveolates, Apicomplexa

Rhizaria, Endomixa

Excavates, Euglenozoa

Excavates, Heterolobosea

Excavates, Heterolobosea

Excavates, Fornicata

Excavates, Fornicata

Excavates, Parabasalia

Haptophyta, Isochrysidales

Haptophyta, Prymnesiales

Haptophyta, Pavlovales

Haptophyta, Phaeocystales
Y

$Y$

$\mathrm{N}$

$Y$

Y

$\mathrm{N}$

$\mathrm{N}$

$Y$

Y

$\mathrm{N}$

$\mathrm{N}$

N

$\mathrm{N}$

$\mathrm{N}$

$\mathrm{N}$

N

$\mathrm{N}$

$\mathrm{N}$

$\mathrm{N}$

$\mathrm{N}$

N

$\mathrm{N}$

$\begin{array}{ll}\mathrm{Y} & \mathrm{Y}\end{array}$

$\mathrm{N} \quad \mathrm{N}$

Y N

Y N 